\title{
Signatures of ALOS PALSAR L-Band Backscatter in Swedish Forest
}

\author{
Maurizio Santoro, Member, IEEE, Johan E. S. Fransson, Member, IEEE, Leif E. B. Eriksson, Member, IEEE, \\ Mattias Magnusson, Lars M. H. Ulander, Senior Member, IEEE, and Håkan Olsson
}

\begin{abstract}
The Phased Array type L-band Synthetic Aperture Radar onboard the Advanced Land Observing Satellite has, since its launch, been acquiring an extensive data set of images over two forest test sites in Sweden. The sites of Remningstorp (Lat. $58^{\circ} 30^{\prime} \mathrm{N}$, Long. $13^{\circ} 40^{\prime} \mathrm{E}$ ) in the south and Krycklan (Lat. $64^{\circ} 14^{\prime} \mathrm{N}$, Long. $19^{\circ} 50^{\prime} \mathrm{E}$ ) in the north of Sweden are characterized by hemiboreal and boreal forests, respectively. In this paper, we have investigated the signatures of standwise backscatter measurements from forests with different growth stages in relation to polarization, environmental conditions, image viewing geometry, and spatial resolution. The HV backscatter presented stronger sensitivity to the forest growth stage than the $\mathrm{HH}$ and VV backscatter. Under unfrozen conditions, the dynamic range of fine-beam data acquired at $34.3^{\circ}$ was 8-9 dB for the $\mathrm{HV}$ polarization and 6-7 $\mathrm{dB}$ for the $\mathrm{HH}$ polarization. At $21.5^{\circ}$, in the polarimetric mode, the dynamic range was 6,7 , and $9 \mathrm{~dB}$ at VV, HH, and HV polarizations, respectively. Regardless of the specific polarization, the backscatter was temporally consistent under unfrozen conditions, with a small increase of backscatter in regrowing young forest for wet conditions. Under thawing and frozen conditions, repeated measurements were available only for the HH backscatter at $34.3^{\circ}$. For thawing conditions, the backscatter level was similar to the unfrozen conditions even though the signatures differed depending on temperature dynamics, snow-cover properties, and precipitation. Under frozen conditions, the signatures varied depending on temperature. For images acquired when the temperature was well below the freezing point, the backscatter was low, and the dynamic range was small (2-4 dB); nonetheless, the measurements were consistent. Images acquired when temperature was close to the freezing point presented a behavior similar to unfrozen conditions. The sensitivity of the backscatter to the image viewing geometry for different growth stages was studied for data acquired under dry unfrozen conditions. The backscatter difference increased for increasing look angle because of the increase in volume scattering and the decrease of ground-surface backscatter. The largest difference was observed at $41.5^{\circ}$ with $2.5-4-\mathrm{dB}$ difference for the $\mathrm{HH}$ and 4-5-dB
\end{abstract}

Manuscript received December 12, 2008; revised March 26, 2009 and April 20, 2009. First published August 18, 2009; current version published November 25, 2009. This work was supported in part by the Swedish National Space Board and in part by Hildur and Sven Wingquist's Foundation for Forest Research.

M. Santoro is with Gamma Remote Sensing AG, 3073 Gümligen, Switzerland (e-mail: santoro@gamma-rs.ch).

J. E. S. Fransson and H. Olsson are with the Department of Forest Resource Management, Swedish University of Agricultural Sciences, 90183 Umeå, Sweden (e-mail: Johan.Fransson@ srh.slu.se; Hakan.Olsson@ srh.slu.se).

L. E. B. Eriksson and L. M. H. Ulander are with the Department of Radio and Space Science, Chalmers University of Technology, 41296 Gothenburg, Sweden (e-mail: leif.eriksson@chalmers.se; ulander@foi.se).

M. Magnusson was with the Department of Forest Resource Management, Swedish University of Agricultural Sciences, 90183 Umeå, Sweden. He is now with Växjö Stift, 35106 Växjö, Sweden (e-mail: Magnusson@srh.slu.se).

Color versions of one or more of the figures in this paper are available online at http://ieeexplore.ieee.org.

Digital Object Identifier 10.1109/TGRS.2009.2023906 difference for the HV case. Loss of spatial resolution (20-50 m) did not have any effect on the backscatter signatures in Krycklan, whereas in Remningstorp, the smallest stands were affected.

Index Terms-Advanced Land Observing Satellite (ALOS) Phased Array type L-band Synthetic Aperture Radar (PALSAR), boreal forest, environmental conditions, image viewing geometry, polarization, SAR backscatter, Sweden.

\section{INTRODUCTION}

$\mathbf{T}$ HE PHASED Array type L-band Synthetic Aperture Radar (PALSAR) on the Advanced Land Observing Satellite (ALOS) is a multiconfiguration system capable of acquiring images in single, dual, and quad polarization using the Stripmap mode and in single polarization using the ScanSAR mode. Despite the large number of possible imaging configurations, ALOS PALSAR is operated in three Stripmap configurations and one ScanSAR configuration following a systematic image-acquisition strategy. The acquisition plan was developed before the launch of the satellite to avoid operational conflicts, to build up a consistent data archive, and to attempt maximizing the information content of the data with respect to the applications targeted with the satellite [1]. Previous investigations contributed to the definition of the acquisition plan.

For boreal and hemiboreal forests, the L-band copolarized and cross-polarized backscatter show dependence on the growth stage and, thus, on biophysical parameters [2]-[19]. All these investigations showed that the backscatter is low in treeless or practically unvegetated areas such as clear-cuts or burnt areas. It tends to increase with increasing canopy cover and density and size of the trees, reaching a saturation level, i.e., loss of sensitivity to forest biophysical parameters such as biomass or stem volume, in denser forests. The sensitivity of the backscatter to forest parameters and the saturation level seem to be rather site dependent, since forest structure influences the relative contribution of the scattering mechanisms. In addition, the individual contributions to the total forest backscatter depend on the environmental conditions (i.e., weather conditions, moisture conditions, and weather dynamics), which affect the dielectric properties of the vegetation and the ground surface.

Investigations on the backscatter properties of boreal and hemiboreal forests at L-band identified the dominant scattering mechanism in the direct scattering from the canopy [3], [7], [11], [19]-[21]. Some authors indicated more specifically the origin to be at the level of the large branches [2], [13]. Trunkground interactions were found to be negligible in [2] and [19], with some exceptions at $\mathrm{HH}$ polarization [7], for sparser or young forests [11], for some specific structural properties 
[13], [20], or under frozen environmental conditions [22]. The direct surface scattering at $\mathrm{HH}$ and $\mathrm{VV}$ polarizations represents a small part of the total backscatter [3], [11], [13] but can increase for wet conditions of the ground surface [14], [15], [18], [23], [24]. For cross polarization, the direct surface component to the total backscatter is negligible and unaffected by the ground conditions [3], [7], [24]. As a consequence, crosspolarized backscatter data from boreal and hemiboreal forests show larger dynamic range, i.e., backscatter difference between unvegetated forest areas and dense forests, compared to copolarized backscatter [2]-[4], [7], [9], [10], [14], [15], [19]. The higher reflectivity of HH-polarized waves as compared to VV-polarized waves explains why several authors noticed a slightly higher sensitivity of HH backscatter than VV backscatter to forest properties [2], [10], [15], [19], [25].

Over the northern regions, ALOS PALSAR acquires polarimetric data primarily in the fine-beam dual (FBD) mode, in $\mathrm{HH}$ and $\mathrm{HV}$ polarizations, and along two or three repeat-pass cycles per year in summer and fall. In the polarimetric (PLR) mode, all four polarizations are recorded. However, PLR images are only acquired locally and along one or two repeat-pass cycles per year outside the winter season [1]. During winter, the frozen conditions make the forest canopy more transparent so that the sensitivity of the backscatter to forest properties decreases [4], [13], [14], [17], [18], [22], [26]. For this reason, PALSAR acquires only at one polarization, $\mathrm{HH}$, in the $\mathrm{FB}$ single (FBS) mode.

At present, the Swedish Forest Agency is annually acquiring a nationwide set of optical Satellite Pour l'Observation de la Terre (SPOT) satellite data, which is primarily used for manual verification of about 70000 cutting permits granted among more than 300000 land owners. In addition, the Swedish University of Agricultural Sciences (SLU) is producing nationwide forest stem-volume maps, using SPOT satellite data trained with about 50000 National Forest Inventory field plots. Sweden is however characterized by frequent cloud cover and long periods of reduced solar illumination. In this respect, spaceborne SAR is of particular interest as a future complement for forest monitoring. Since SAR is independent of cloud cover and day/night cycle, multiple acquisitions over relatively short periods of time can be guaranteed. The sensitivity of the backscattered signal at L-band to forest structural properties motivates further investigation of the usefulness of ALOS PALSAR images for forest mapping and change detection.

Starting in May 2006, i.e., four months after the launch of ALOS, PALSAR has repeatedly acquired images over a number of test sites, owing to the support from the ALOS Calibration and Validation activities [27] and the participation in the Japan Aerospace Exploration Agency (JAXA) Kyoto \& Carbon (K\&C) Initiative [1], [28]. Since then, a large data set of images acquired in different modes, image viewing geometries, and under several environmental conditions has been gathered (see Section III for details). Such variety has enabled investigations on the signatures of PALSAR L-band SAR backscatter with the aim of setting up or improving methods for forest mapping and change detection. Knowledge about the factors affecting the PALSAR backscatter helps in defining the applica-

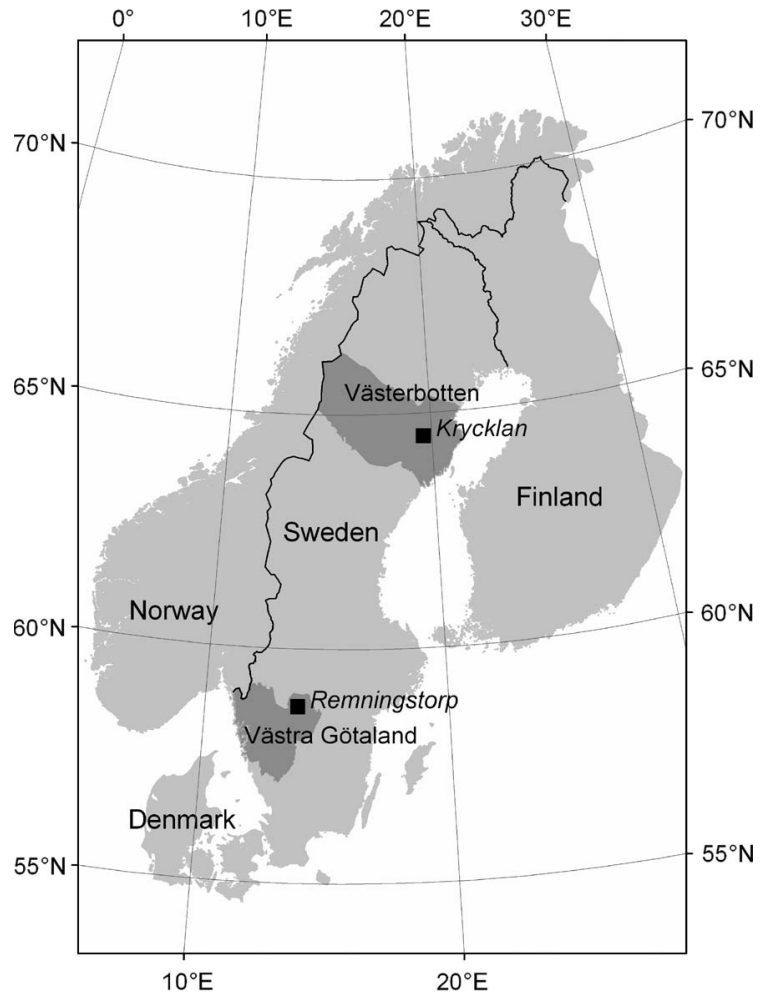

Fig. 1. Map of test sites and corresponding counties.

tions that might be feasible and whether specific polarizations, environmental conditions, or image viewing geometries are more favorable than others for a given application.

In this paper, we present a signature analysis of the ALOS PALSAR L-band backscatter with respect to polarization, environmental conditions, and viewing geometry. In addition, the availability of SAR images processed at different pixel sizes allows the studying of the effect of spatial resolution on the forest backscatter signatures. The analysis has been carried out for different forest growth stages, ranging between regrowing young forest and dense mature forest, and over two test sites in northern and southern Sweden. In this way, the effect of structural properties of the forests could also be investigated. Section II presents an overview of the test sites and the in situ measurements. The PALSAR data set is presented in Section III. In Section IV, results from the signature analysis are discussed. The conclusion and an outlook concerning future investigations are presented in Section V.

\section{Test Sites And IN SITU DatA}

As test sites, we considered the forest holding of Remningstorp (Lat. $58^{\circ} 30^{\prime} \mathrm{N}$, Long. $13^{\circ} 40^{\prime} \mathrm{E}$ ) in the south of Sweden and the forest area of Krycklan (Lat. $64^{\circ} 14^{\prime}$ N, Long. $19^{\circ} 50^{\prime} \mathrm{E}$ ) in the north of Sweden (Fig. 1). Remningstorp, located in the county of Västra Götaland, is characterized by hemiboreal forest, thus also including forest with high stem volume. Krycklan is located within the boreal zone in the county of Västerbotten. Forest cover is somewhat sparser, and stem volumes are lower as compared to Remningstorp (see Table I and Fig. 2). These aspects will be discussed in more detail in the following sections dedicated to each test site. 
TABLE I

STAND-Size AND STEM-Volume STATistics For THE USED STANDS AT THE TEST Sites

\begin{tabular}{lll}
\hline & Remningstorp & Krycklan \\
\hline Number of forest stands & 559 & 509 \\
\hline Stand size (min-max) & $0.1-20.6 \mathrm{ha}$ & $1.2-25.2 \mathrm{ha}$ \\
\hline Mean stand size & $2.5 \mathrm{ha}$ & $7.5 \mathrm{ha}$ \\
\hline Stem volume (min-max) & $0-694 \mathrm{~m} / \mathrm{ha}$ & $1-270 \mathrm{~m}^{3} / \mathrm{ha}$ \\
\hline Mean stem volume & $192 \mathrm{~m}^{3} / \mathrm{ha}$ & $107 \mathrm{~m}^{3} / \mathrm{ha}$ \\
\hline
\end{tabular}

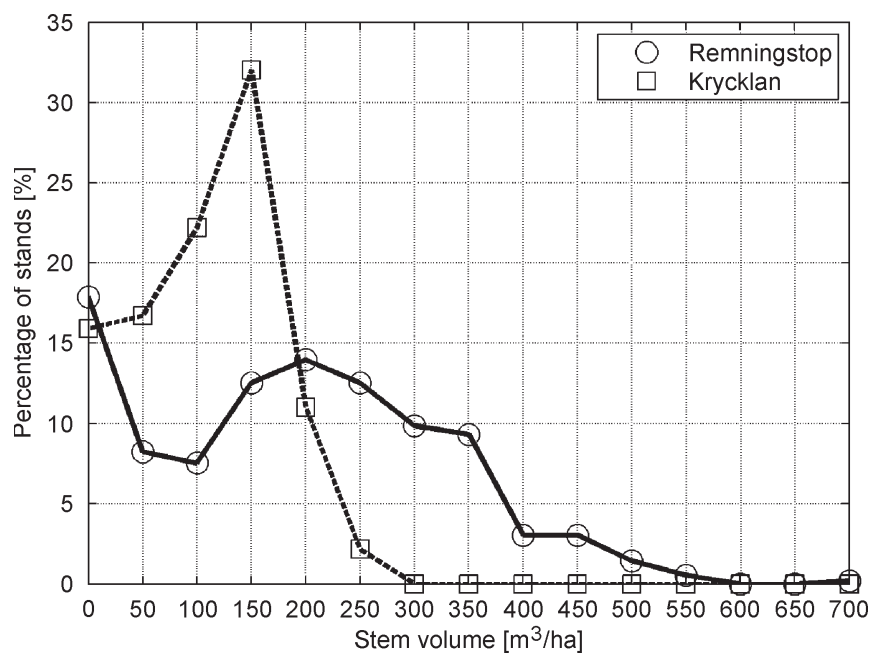

Fig. 2. Distribution of stem volume for the used stands in Remningstorp and Krycklan. Stands have been grouped in classes being $50 \mathrm{~m}^{3} /$ ha wide and centered in the values reported on the $x$-axis.

\section{A. Remningstorp Test Site}

The Remningstorp test site covers about 1200 ha of productive forestland. The prevailing tree species are Norway spruce (Picea abies) and Scots pine (Pinus sylvestris), followed by birch (Betula spp.). The dominant soil type is till (i.e., a mixture of glacial debris) with a field layer consisting of different herbs, blueberry (Vaccinium myrtillus), and narrow-leaf grass (e.g., Deschampsia flexuosa). In denser old spruce stands, the field layer is absent. The ground topography is mainly flat with elevation varying between 120 and $145 \mathrm{~m}$ a.s.l.

The data consisted of a digital stand-boundary map in vector format and in situ measurements of several forest variables (e.g., stem volume and tree-species composition). Stand boundaries were delineated from data acquired by a digital aerial camera (Z/I DMC) during an airborne campaign flown in the fall of 2003. The delineation was performed using aerial photointerpretation on a digital photogrammetric workstation. Aerial photointerpretation was also used for the estimation of standwise forest variables using film-based panchromatic photographs with a scale of $1: 30000$ acquired during 2004. The stand delineation and forest-variable estimation were updated with support of ortophotographs from 2006 and a subjective field inventory completed during the summer of 2008. In total, 662 stands were delineated, of which 559 were labeled as forest stands. For the year of 2008, statistics on stand size and stem volume are given in Table I, and the distribution of stem volume is shown in Fig. 2. All forest growth stages, ranging between regrowing young forest and dense mature forest, were well represented. Stem volume was almost uniformly distributed between 0 and $400 \mathrm{~m}^{3} /$ ha, with several stands above this level up to $700 \mathrm{~m}^{3} /$ ha. The root mean-square error (rmse) for the stem-volume estimation using aerial photointerpretation is about 20\%-25\% [29].

For this investigation, the digital stand-boundary map was converted to raster format with a pixel spacing of $20 \mathrm{~m}$. Edge erosion, which can be performed to reduce the risk for geolocation errors and edge effects on the standwise backscatter signatures, was not applied to avoid decreasing the size of the stands significantly.

\section{B. Krycklan Test Site}

The Krycklan test site includes 6800 ha of mainly forested land. Topography is hilly with several gorges, and the ground elevation ranges between 125 and $350 \mathrm{~m}$ a.s.l. A valley crosses the southern part of the site, and to the north is a hilly area with ridges aligned approximately NW-SE. Most of the area is covered by forest, with some scattered agricultural fields, wetlands, and lakes. In the forested areas, the dominant soil type is till. The mean ground slope of most stands considered in this paper was less than $5^{\circ}$. For $90 \%$ of the stands, the ground slope was less than $10^{\circ}$, and $98 \%$ of the stands had a ground slope less than $15^{\circ}$.

A forest inventory was carried out in the end of May 2007. This involved a systematic sample of 131 plots of 7-m radius across the whole test site. Of these, $65 \%$ were pine dominated, $25 \%$ spruce dominated, and $10 \%$ were dominated by deciduous trees, mainly birch. At plot level, stem volumes were in the range of 1-408 $\mathrm{m}^{3} / \mathrm{ha}$, with an average of $127 \mathrm{~m}^{3} / \mathrm{ha}$. The Krycklan test site was also laser scanned on September 9, 2006 using the ALTM 3100C Optech airborne laser instrument operated by Blom Geomatics AS, Norway. The laser scanning took place from an altitude of $1.1 \mathrm{~km}$, resulting in a pulse density of 3-4 pulses $/ \mathrm{m}^{2}$. A segmentation of the forested area was initially performed to obtain the stand boundaries. The segmentation was based on the laser-scanning data and optical SPOT-5 satellite data in combination. In total, 509 stands were created. Regression analysis was applied to establish the relationship between stem volume estimated from field measurements and laser-derived height together with canopy density at plot level [30], [31]. Using the regression function, stem volume was estimated for a raster $(10 \mathrm{~m} \times 10 \mathrm{~m})$ covering the test site. These estimates were then aggregated to stand level.

For this investigation, the forest stands were edge eroded. Because of the rather large size of the forest stands, a 20-mwide buffer zone, corresponding to the pixel size of the stand map, was removed from the perimeter of each forest stand. Statistics on stand size and stem volume are given in Table I. The distribution of stem volume for the 509 stands is shown in Fig. 2. As for Remningstorp, all growth stages are well represented. As compared to Remningstorp, the distribution of stem volume is clearly toward smaller stem volumes, with a peak at around $150 \mathrm{~m}^{3} /$ ha that, however, corresponds to a relatively high stem volume at the latitude of this test site. The rmse for the stem-volume estimation was found to be about $20 \%-30 \%$. 


\section{PALSAR DATA SeT}

For each test site, the PALSAR data set consisted primarily of path images, i.e., images in full resolution, acquired in FBS, FBD, and PLR modes at different look angles $\left(21.5^{\circ}\right.$, $34.3^{\circ}, 41.5^{\circ}$, and $50.8^{\circ}$ ). A SAR data set was also available at a reduced spatial resolution in a special format produced by JAXA. These images will be referred to as K\&C PALSAR data strips, since they are long strips prepared and delivered by JAXA in the framework of the K\&C Initiative [1]. In the following, a combined notation of acquisition mode and look angle will be used to refer to a specific imaging configuration. For example, FBD34 is used for images acquired in FBD with a look angle of $34.3^{\circ}$.

\section{A. Data Set of Path Images}

Table IV summarizes the number of data sets available for each mode. An atypical feature of the data sets is the availability of a relatively large number of images in FB mode acquired in geometries different than the standard one $\left(34.3^{\circ}\right.$ along ascending orbits).

Path images were obtained in single-look complex format, from which calibrated multilooked SAR backscatter images were generated. Multilook factors for the range and the azimuth direction depended on the imaging configuration $(2 \times 6$ for FBS21, $2 \times 5$ for FBS34, $2 \times 4$ for FBS41, $2 \times 8$ for FBD34, $2 \times 7$ for FBD41 and FBD50, and $1 \times 7$ for PLR21 images). In all cases, the aim was to remove speckle without losing too much resolution. The pixel size of the multilook intensity images in the radar geometry was about 20-25 m. Further reduction of speckle noise was not applied, since this data set was primarily meant for the computation of standwise averages of the backscatter. The updated calibration factors provided by JAXA were used for absolute calibration [32]. For image geocoding, a digital elevation model (DEM) acquired from the Swedish National Land Survey with 50-m posting was used. The DEM was oversampled to allow geocoding of the SAR images to 20-m pixel size. Geocoding of the SAR data was based on a lookup table describing the transformation between the radar and the map geometry [33], [34]. The lookup table is generated on the basis of orbital information and elevation in the DEM. Typically, a refinement of the lookup table is required because of inaccuracies in the orbital information. The refinement is implemented in the form of offsets estimation between the SAR image to be geocoded and a reference image (e.g., a simulated SAR image from the DEM) transformed to the radar geometry. The two images are divided in a number of image chips, and offsets between corresponding chips are estimated using a cross-correlation algorithm. The image chips are distributed homogeneously on the image data, with number and size being strictly dependent on the correlating features between the SAR image and the reference image. A linear polynomial is estimated from the offsets and applied to refine the lookup table. Quality of the refinement is given by the rmse between measured and estimated offsets using the offset polynomial. This is basically a measure of the correspondence between the two images, i.e., the geocoding error with respect to the reference geometry. The error level depends on the amount of topographic features within the image scene; higher error occurs for images covering predominantly flat areas (e.g., in desert areas of floodplains). In our case, the rmse was between 0.2 and 0.4 times the pixel size in the radar geometry, i.e., approximately 5-10 m. The SAR images are finally transformed to the map geometry using the refined lookup table. To correct for possible offsets between images acquired along different orbital tracks, fine coregistration was applied using the cross-correlation algorithm. Coregistration errors were less than $1 / 5$ th of the pixel size in the map geometry, i.e., $4 \mathrm{~m}$, thus indicating overall satisfactory geocoding of the path images.

The geocoded SAR images presented topographic effects on the backscatter in areas of sloped terrain. These effects were particularly visible at the Krycklan test sites because of the hilly terrain. The image on the left-hand side of Fig. 3 shows the subset of a geocoded SAR image covering the test site of Krycklan in sigma-nought $\sigma^{0}$ format. Several slopes facing the radar appear brighter with an enhanced backscatter. For a correct interpretation of the backscatter signatures, correction for the effect of local incidence angle and normalization for the true pixel area were necessary [35]. It was shown in [36] that uncompensated topographic effects induce a $2-7-\mathrm{dB}$ dispersion of the L-band backscatter, which is a drawback when assessing the properties of the backscatter because, at this frequency, the forest/non-forest contrast is of the same order.

The corrected backscatter in gamma-nought $\gamma^{0}$ format can be obtained from the sigma-nought $\sigma^{0}$ value according to [35], [36]

$$
\gamma^{0}=\sigma^{0} \frac{A_{\text {flat }}}{A_{\text {slope }}}\left(\frac{\cos \theta_{\text {ref }}}{\cos \theta_{\text {loc }}}\right)^{n}
$$

where $\theta_{\text {loc }}$ and $\theta_{\text {ref }}$ represent the local incidence angle and a reference angle for the normalization of the backscatter (e.g., the incidence angle at midswath), respectively. $A_{\text {slope }}$ and $A_{\text {flat }}$ represent the true pixel area and the local pixel area for a theoretically flat terrain, respectively. For bare surfaces, the exponent $n$ is equal to one. For vegetated surfaces, it expresses the variation of the scattering mechanism due to the presence of a volume on the sloped terrain, thus being related to the optical depth of the vegetation. At the forest site of Lozère, France, the value was estimated between 0.75 and 0.91 for AIRSAR and Shuttle Imaging Radar SIR-C L-band SAR backscatter, respectively. For the Japanese Earth Resources Satellite (JERS)-1 L-band SAR backscatter, the value was lower and equal to 0.36 [36]. Considering that $n=1$ corresponds to the case of a bare surface, the additional correction is relevant for the JERS-1 configuration (HH polarization and $35^{\circ}$ look angle). PALSAR operates in standard FB modes at $34.3^{\circ}$ look angle, thus implying that a correction that also takes into account the scattering properties of the forest should have been applied. The lack of detailed information about optical depth of the forest canopies at the test sites did not allow us to determine a suitable value for $n$ so that $n=1$ was used. The implications will be discussed in Section IV-C. The image on the right-hand side of Fig. 3 shows the backscatter after compensation for topographic effects. The gamma-nought image appeared more uniform as compared to the original image in sigma-nought format.

Fig. 4 shows a subset covering the Remningstorp test site obtained from the FBD34 HV-polarized image acquired on 

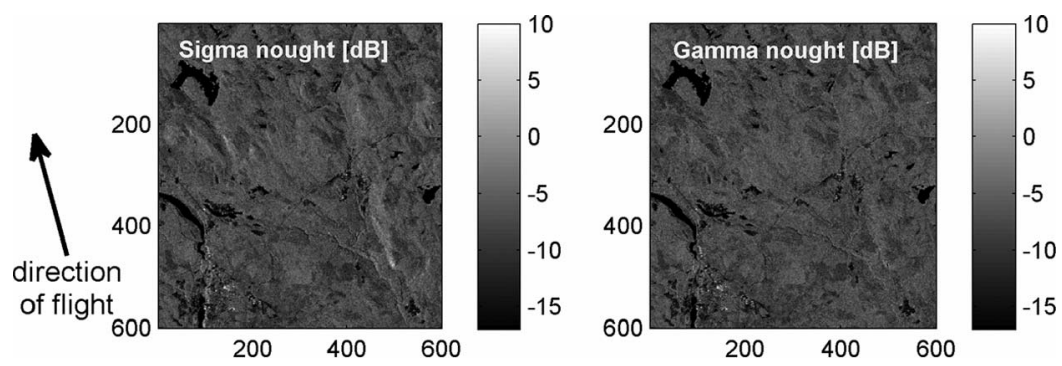

Fig. 3. Subset covering the Krycklan test site of the FBD34 HH-polarized PALSAR image acquired on July 7, 2007 (left) before and (right) after correction for local incidence angle and pixel-area effects. Each image is 600 pixels wide and long, corresponding to $12 \mathrm{~km}$ in easting and northing.

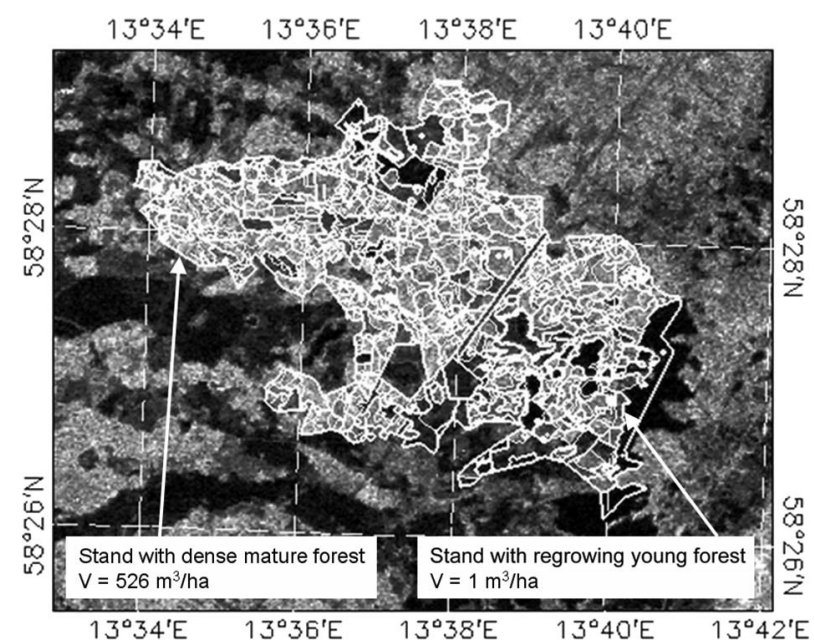

Fig. 4. FBD34 HV gamma-nought image acquired on August 1, 2007 over the Remningstorp test site overlaid with the forest-stand boundaries. The area covered by this image is $9 \mathrm{~km}$ wide and $7 \mathrm{~km}$ long.

August 1, 2007. The boundaries of the stands have been overlaid. High backscatter corresponds to forests; somewhat lower backscatter within the test site corresponds to regrowing young forest. The areas on the western side of the image with low backscatter correspond to bare agricultural fields. Toward the eastern part of the test site, several lakes can be distinguished because of the very low backscatter. Fig. 5 shows an analogous example for the Krycklan test site. The subset has been obtained from the HV-polarized image acquired on August 22, 2007 in FBD mode at $34.3^{\circ}$ look angle. The area is predominantly covered with forests, whereas clear-cuts show lower backscatter. Very low backscatter corresponds to lakes, rivers, and a few agricultural fields.

\section{B. Data Set of $K \& C$ Strip Images}

Within the $\mathrm{K} \& \mathrm{C}$ Initiative, multilooked amplitude images with approximately $50-\mathrm{m}$ pixel spacing along the azimuth direction have been provided by JAXA. K\&C image strips acquired over Sweden are intended for mapping of clear-felled areas and forest resources in the counties of Västerbotten and Västra Götaland (see Fig. 1 and [28]). Each year, one cycle of images acquired in FBS34 mode during winter and two cycles of images acquired in FBD34 mode during summer/fall have been delivered. In this paper, standwise backscatter measurements from $\mathrm{K} \& \mathrm{C}$ image strips covering the two test sites have been compared to the corresponding measurements from the higher resolution path data. In Tables II and III, acquisition

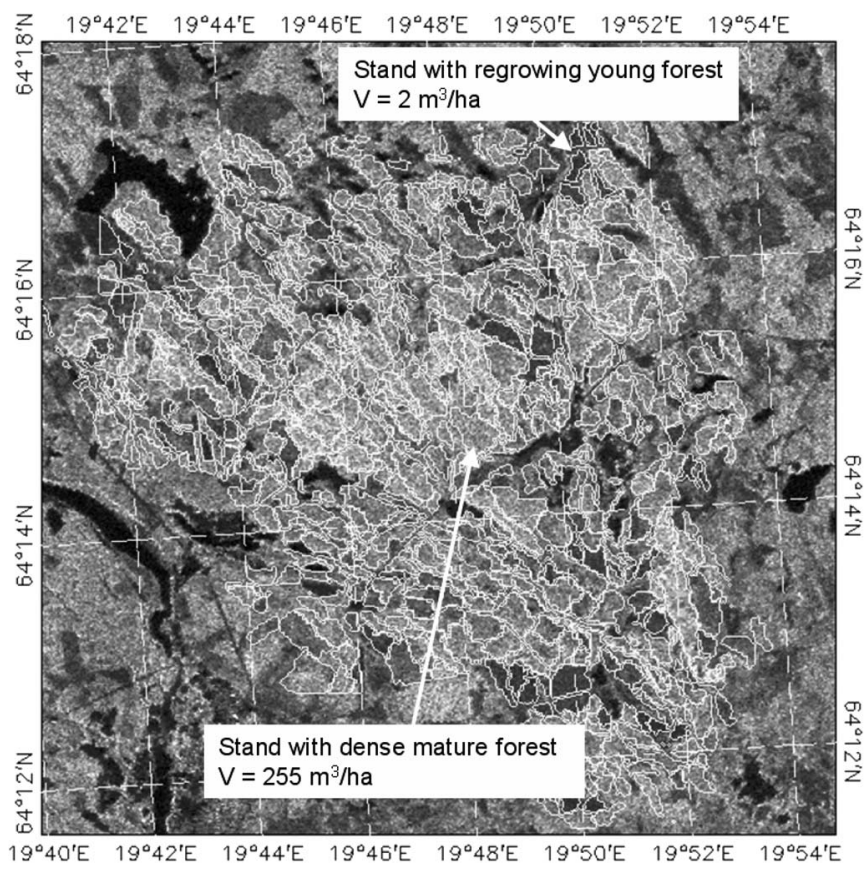

Fig. 5. FBD34 HV gamma-nought image acquired on August 22, 2007 over the Krycklan test site overlaid with the forest-stand boundaries. The area covered by this image is $12 \mathrm{~km}$ wide and $12 \mathrm{~km}$ long.

dates for which both path images and $\mathrm{K} \& \mathrm{C}$ image strips were available are indicated with an asterisk.

Processing of the $\mathrm{K} \& \mathrm{C}$ image strips consisted of conversion to intensity, absolute calibration, terrain geocoding, and topographic correction of the backscatter. Since the image had already been strongly multilooked by JAXA (multilook factors 4 and 16), further speckle reduction was a minor issue. Multilook of two pixels in range was applied to FBS images to obtain the same pixel size as for FBD data, i.e., approximately $60 \mathrm{~m}$. The updated calibration factors provided by JAXA were used for absolute calibration [32]. All strips were geocoded to 50-m pixel size in order to adhere to the pixel size in the radar geometry. The DEM and the geocoding procedure used were the same as for the path images. The rmse between measured and estimated offsets based on the offset polynomial, i.e., the geocoding error, was below $1 / 3$ of the pixel size in the SAR geometry, i.e., approximately $15 \mathrm{~m}$. To increase the geolocation accuracy, coregistration of data strips from the same reference system for planning (RSP), as well as between adjacent data strips, was applied. This was possible owing to the strong swath overlap of adjacent tracks $(\sim 50 \%)$ at the latitudes of the 
TABLE II

ALOS PAlSAR Data Sets in Terms of Acquisition Date, ImAging Configuration, RSP Number, Flight Direction

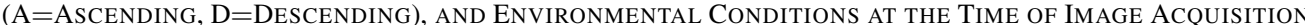

$(F R=$ FroZEN, UNFR $=$ UnFrozen, THAW $=$ THAWED CONDITIONS $)$. TEST SITE: REMNINGSTORP. DATA SETS Marked With an Asterisk Were Available Both as Path Images and K\&C Strip Images

\begin{tabular}{|c|c|c|c|c|c|c|}
\hline ID & $\begin{array}{c}\text { Acquisition } \\
\text { date }\end{array}$ & $\begin{array}{l}\text { Imaging } \\
\text { config. }\end{array}$ & $\begin{array}{c}\text { RSP } \\
\text { number }\end{array}$ & $\begin{array}{c}\text { Flight } \\
\text { direction }\end{array}$ & Environmental conditions & \\
\hline 1 & $2006-05-20$ & PLR21 & 311 & $\mathrm{D}$ & $\mathrm{T} \approx 9^{\circ} \mathrm{C}, 5 \mathrm{~mm}$ precipitation & \multirow{24}{*}{ Unfr } \\
\hline 2 & $2006-05-25$ & FBS41 & 636 & A & $T \approx 10^{\circ} \mathrm{C}$, dry & \\
\hline 3 & $2006-06-03$ & PLR21 & 623 & A & $\mathrm{T} \approx 14^{\circ} \mathrm{C}$, dry & \\
\hline 4 & $2006-06-08$ & FBS34 & 304 & $\mathrm{D}$ & $T \approx 12^{\circ} \mathrm{C}$, dry & \\
\hline 5 & $2006-06-23$ & FBD41 & 635 & A & $\begin{array}{l}\mathrm{T} \approx 14 \stackrel{\circ}{\circ} \mathrm{C}, 2 \mathrm{~mm} \text { precipitation, } \\
\text { fourth day of precipitations }\end{array}$ & \\
\hline 6 & $2006-07-05$ & PLR21 & 311 & $\mathrm{D}$ & $\mathrm{T} \approx 19^{\circ} \mathrm{C}, \mathrm{dry}$ & \\
\hline 7 & $2006-07-07$ & FBS34 & 303 & $\mathrm{D}$ & $T \approx 21^{\circ} \mathrm{C}$, dry & \\
\hline 8 & $2006-07-10$ & FBS41 & 636 & A & $\mathrm{T} \approx 20^{\circ} \mathrm{C},<1 \mathrm{~mm}$ precipitation & \\
\hline 9 & $2006-07-19$ & PLR21 & 623 & A & $T \approx 21^{\circ} \mathrm{C}$, dry & \\
\hline 10 & $2006-08-03$ & FBS21 & 310 & D & $\begin{array}{l}\mathrm{T} \approx 15^{\circ} \mathrm{C}, 3 \mathrm{~mm} \text { precipitation, } \\
\text { fourth day of precipitations }\end{array}$ & \\
\hline 11 & $2006-08-08$ & FBD41 & 635 & A & $\mathrm{T} \approx 21^{\circ} \mathrm{C},<1 \mathrm{~mm}$ precipitation & \\
\hline 12 & $2006-08-20$ & PLR21 & 311 & D & $\mathrm{T} \approx 15^{\circ} \mathrm{C}, 6 \mathrm{~mm}$ precipitation & \\
\hline 13 & 2006-09-03 & PLR21 & 623 & A & $\begin{array}{l}\mathrm{T} \approx 15^{\circ} \mathrm{C}, 1 \mathrm{~mm} \text { precipitation, } \\
\text { third day of precipitations }\end{array}$ & \\
\hline 14 & $2006-09-08$ & FBS34 & 304 & $\mathrm{D}$ & $\mathrm{T} \approx 9^{\circ} \mathrm{C}$, dry, two days of precipitations before & \\
\hline 15 & $2006-09-18$ & FBS21 & 310 & $\mathrm{D}$ & $\mathrm{T} \approx 11^{\circ} \mathrm{C},<1 \mathrm{~mm}$ precipitation & \\
\hline 16 & $2006-10-05$ & PLR21 & 311 & $\mathrm{D}$ & $\mathrm{T} \approx 9^{\circ} \mathrm{C},<1 \mathrm{~mm}$ precipitation & \\
\hline 17 & $2006-10-07$ & FBS34 & 303 & $\mathrm{D}$ & $\mathrm{T} \approx 14^{\circ} \mathrm{C},<1 \mathrm{~mm}$ precipitation & \\
\hline 18 & $2006-10-10$ & FBD41 & 636 & A & $T \approx 12^{\circ} \mathrm{C}, \mathrm{dry}$ & \\
\hline 19 & $2006-10-19$ & PLR21 & 623 & A & $\mathrm{T} \approx 10^{\circ} \mathrm{C},<1 \mathrm{~mm}$ precipitation & \\
\hline 20 & $2006-11-08$ & FBS41 & 635 & A & $\mathrm{T} \approx 6{ }^{\circ} \mathrm{C}, 4 \mathrm{~mm}$ precipitation & \\
\hline 21 & $2006-11-14$ & FBD50 & 289 & D & $\mathrm{T} \approx 4^{\circ} \mathrm{C}, 1 \mathrm{~mm}$ precipitation & \\
\hline 22 & 2006-12-04 & PLR21 & 623 & A & $\begin{array}{l}\mathrm{T} \approx 6{ }^{\circ} \mathrm{C}, 1 \mathrm{~mm} \text { precipitation, } \\
\text { fourth day of precipitations }\end{array}$ & \\
\hline 23 & 2006-12-30 & FBD50 & 289 & D & $\begin{array}{l}\mathrm{T} \approx 3^{\circ} \mathrm{C}, 3 \mathrm{~mm} \text { precipitation, } \\
\text { fourth day of precipitations }\end{array}$ & \\
\hline 24 & $2006-12-31$ & FBS34 & 630 & A & $\begin{array}{l}\mathrm{T} \approx 7^{\circ} \mathrm{C}, 3 \mathrm{~mm} \text { precipitation, } \\
\text { fifth day of precipitations }\end{array}$ & \\
\hline $25^{*}$ & $2007-01-29$ & FBS34 & 629 & $\mathrm{~A}$ & $\mathrm{~T} \approx-5^{\circ} \mathrm{C}$, dry, snow depth: $6 \mathrm{~cm}$ & \multirow{2}{*}{$\mathrm{Fr}$} \\
\hline $26^{*}$ & $2007-02-15$ & FBS34 & 630 & A & $\mathrm{T} \approx-3^{\circ} \mathrm{C}$, dry, snow depth: $3 \mathrm{~cm}$ & \\
\hline 27 & $2007-04-21$ & PLR21 & 623 & A & $\mathrm{T} \approx 5 \stackrel{\circ}{\mathrm{C}}, \mathrm{dry}$ & \multirow{11}{*}{ Unfr } \\
\hline 28 & $2007-06-06$ & PLR21 & 623 & A & $T \approx 23^{\circ} \mathrm{C}$, dry & \\
\hline 29 & $2007-06-16$ & FBD34 & 629 & A & $\mathrm{T} \approx 14^{\circ} \mathrm{C}, 14 \mathrm{~mm}$ precipitation & \\
\hline 30 & $2007-07-03$ & FBD34 & 630 & A & $\mathrm{T} \approx 14^{\circ} \mathrm{C}, 37 \mathrm{~mm}$ precipitation & \\
\hline $31^{*}$ & $2007-08-01$ & FBD34 & 629 & A & $T \approx 16^{\circ} \mathrm{C}$, dry & \\
\hline $32^{*}$ & $2007-08-18$ & FBD34 & 630 & A & $\mathrm{T} \approx 13^{\circ} \mathrm{C}, 2 \mathrm{~mm}$ precipitation & \\
\hline $33^{*}$ & $2007-09-16$ & FBD34 & 629 & A & $\mathrm{T} \approx 13^{\circ} \mathrm{C}, 3 \mathrm{~mm}$ precipitation & \\
\hline $34^{*}$ & $2007-10-03$ & FBD34 & 630 & A & $\mathrm{T} \approx 7^{\circ} \mathrm{C}$, dry, three days of precipitation before & \\
\hline 35 & $2007-11-01$ & FBS34 & 629 & A & $T \approx 3^{\circ} \mathrm{C}, \mathrm{dry}$ & \\
\hline 36 & $2007-11-18$ & FBS34 & 630 & A & $T \approx 4^{\circ} \mathrm{C}$, dry & \\
\hline 37 & $2007-12-07$ & PLR21 & 623 & A & $\begin{array}{l}\mathrm{T} \approx 6^{\circ} \mathrm{C}, 6 \mathrm{~mm} \text { precipitation, } \\
\text { seventh day of precipitations }\end{array}$ & \\
\hline 38 & 2007-12-17 & FBS34 & 629 & A & $\mathrm{T} \approx-2{ }^{\circ} \mathrm{C}$, day/night freeze/thaw cycles & Thaw \\
\hline 39 & 2008-01-03 & FBS34 & 630 & A & $\begin{array}{c}\mathrm{T} \approx-3 \stackrel{\circ}{\circ} \mathrm{C},<1 \mathrm{~mm} \text { precipitation, } \\
\text { snow depth: } 1 \mathrm{~cm}\end{array}$ & $\mathrm{Fr}$ \\
\hline 40 * & $2008-02-01$ & FBS34 & 629 & A & $\begin{array}{l}\mathrm{T} \approx 3 \stackrel{\circ}{\circ} \mathrm{C}, 9 \mathrm{~mm} \text { precipitation, } \\
\text { fourth day of precipitations, } \\
\text { day/night freeze/thaw cycles }\end{array}$ & \multirow[t]{2}{*}{ Thaw } \\
\hline $41^{*}$ & $2008-02-18$ & FBS34 & 630 & A & $\mathrm{T} \approx 3^{\circ} \mathrm{C}$, dry, day/night freeze/thaw cycles & \\
\hline $42^{*}$ & 2008-03-18 & FBS34 & 629 & A & $\begin{array}{l}\mathrm{T} \approx-2 \stackrel{\circ}{ } \mathrm{O}, 11 \mathrm{~mm} \text { precipitation, } \\
\text { snow depth: } 10 \mathrm{~cm}\end{array}$ & $\mathrm{Fr}$ \\
\hline
\end{tabular}

prototype areas. The cross-correlation algorithm already used for the fine registration of the path data was applied. Coregistration errors were below $1 / 20$ th of the pixel size. This confirmed the satisfactory geocoding of the strip images. Finally, the backscatter was corrected for local incidence angle and pixelarea effects based on (1).

Tables II and III give an overview of the PALSAR data sets in terms of acquisition date, imaging configuration, satellite track number (indicated with the acronym RSP), flight direction (ascending, i.e., acquisition at 10:30 A.M., or descending, i.e., acquisition at 10:30 P.M.), and main environmental conditions at the time of image acquisition. The weather data were collected at two weather stations located within the test sites. For Remningstorp, the data set consisted of air temperatures measured daily at 6 A.M. and 6 P.M., maximum and minimum daily air temperatures, daily cumulative precipitation in millimeters, and snow depth in centimeters. Because of the time lag between image acquisition and the temperature measurements, the measurement closest in time to the image acquisition was used. For Krycklan, hourly measurements of air temperature (minimum, maximum, and mean values), daily cumulative precipitation, and weekly snow depth were available. Wind 
TABLE III

ALOS PALSAR Data Sets in Terms of Acquisition Date, ImAging Configuration, RSP Number, Flight Direction ( $\mathrm{A}=$ Ascending, $\mathrm{D}=$ DESCENDING), AND ENVIRONMENTAL CONDITIONS AT THE TIME OF IMAGE ACQUisition

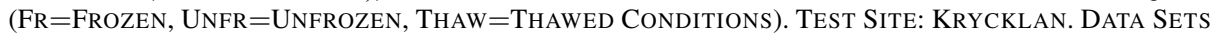
Marked With an Asterisk Were AVailable Both as Path IMAgES AND K\&C STRIP IMAges

\begin{tabular}{|c|c|c|c|c|c|c|}
\hline ID & $\begin{array}{l}\text { Acquisition } \\
\text { date }\end{array}$ & $\begin{array}{l}\text { Imaging } \\
\text { config. }\end{array}$ & $\begin{array}{c}\text { RSP } \\
\text { number }\end{array}$ & $\begin{array}{c}\text { Flight } \\
\text { direction }\end{array}$ & Environmental conditions & \\
\hline 1 & $2006-05-21$ & PLR21 & 606 & A & $\mathrm{T} \approx 3^{\circ} \mathrm{C}$, dry & \multirow{16}{*}{ Unfr } \\
\hline 2 & $2006-05-29$ & FBD41 & 620 & $\mathrm{~A}$ & $\mathrm{~T} \approx 7^{\circ} \mathrm{C}, 10 \mathrm{~mm}$ precipitation & \\
\hline 3 & $2006-06-15$ & FBS41 & 621 & A & $T \approx 10^{\circ} \mathrm{C}, \mathrm{dry}$ & \\
\hline 4 & $2006-06-27$ & FBS34 & 297 & D & $T \approx 17^{\circ} \mathrm{C}, \mathrm{dry}$ & \\
\hline 5 & $2006-06-27$ & FBS41 & 619 & A & $\mathrm{T} \approx 8^{\circ} \mathrm{C}$, dry & \\
\hline 6 & $2006-07-06$ & PLR21 & 606 & A & $\mathrm{T} \approx 15^{\circ} \mathrm{C}, 9 \mathrm{~mm}$ precipitation & \\
\hline 7 & $2006-07-14$ & FBS41 & 620 & A & $\begin{array}{l}\mathrm{T} \approx 10^{\circ} \mathrm{C},<1 \mathrm{~mm} \text { precipitation, } \\
\text { fifth day of precipitations }\end{array}$ & \\
\hline 8 & $2006-07-31$ & FBD41 & 621 & A & $\mathrm{T} \approx 12^{\circ} \mathrm{C}$, dry & \\
\hline 9 & 2006-08-12 & FBS34 & 297 & $\mathrm{D}$ & $\mathrm{T} \approx 24^{\circ} \mathrm{C}, \mathrm{dry}$ & \\
\hline 10 & $2006-08-21$ & PLR21 & 606 & A & $\mathrm{T} \approx 10^{\circ} \mathrm{C}, \mathrm{dry}$ & \\
\hline 11 & $2006-08-27$ & FBS21 & 306 & $\mathrm{D}$ & $T \approx 19^{\circ} \mathrm{C}, \mathrm{dry}$ & \\
\hline 12 & 2006-08-29 & FBD41 & 620 & A & $\begin{array}{l}\mathrm{T} \approx 12^{\circ} \mathrm{C}, 10 \mathrm{~mm} \text { precipitation, } \\
\text { second day of precipitations }\end{array}$ & \\
\hline 13 & $2006-09-15$ & FBS41 & 621 & A & $T \approx 5^{\circ} \mathrm{C}$, dry & \\
\hline 14 & $2006-09-27$ & FBS34 & 297 & D & $\begin{array}{l}\mathrm{T} \approx 9 \stackrel{\circ}{ } \mathrm{C}, 13 \mathrm{~mm} \text { precipitation, } \\
\text { third day of precipitations }\end{array}$ & \\
\hline 15 & $2006-10-12$ & FBS21 & 306 & $\mathrm{D}$ & $\mathrm{T} \approx 5^{\circ} \mathrm{C}$, dry & \\
\hline 16 & $2006-10-14$ & FBS41 & 620 & $\mathrm{~A}$ & $\mathrm{~T} \approx 8 \stackrel{\circ}{ } \mathrm{C}, 4 \mathrm{~mm}$ precipitation & \\
\hline 17 & $2006-11-12$ & FBS34 & 297 & D & $\begin{array}{c}\mathrm{T} \approx 0 \stackrel{\circ}{\circ} \mathrm{C}, 7 \mathrm{~mm} \text { precipitation, } \\
\text { second day of precipitations, } \\
\text { snow depth: } 42 \mathrm{~cm} \text { (on 2006-11-08) and increasing }\end{array}$ & $\mathrm{Fr}$ \\
\hline 18 & $2006-11-21$ & PLR21 & 606 & A & $\begin{array}{c}\mathrm{T} \approx 2{ }^{\circ} \mathrm{C}, 6 \mathrm{~mm} \text { precipitation, } \\
\text { day/night freeze/thaw cycles, } \\
\text { snow depth: } 26 \mathrm{~cm} \text { (on 2006-11-21) and decreasing }\end{array}$ & Thaw \\
\hline 19 & $2006-11-29$ & FBS41 & 620 & A & $\begin{array}{c}\mathrm{T} \approx-2 \stackrel{\circ}{ } \mathrm{C}, 7 \mathrm{~mm} \text { precipitation, } \\
\text { snow depth: } 10 \mathrm{~cm}\end{array}$ & \multirow{5}{*}{$\mathrm{Fr}$} \\
\hline 20 & $2006-12-06$ & FBS34 & 615 & A & $\begin{array}{l}\mathrm{T} \approx-1{ }^{\circ} \mathrm{C}, 4 \mathrm{~mm} \text { precipitation, } \\
\text { fourth day of precipitations, } \\
\text { snow depth: } 1 \mathrm{~cm}\end{array}$ & \\
\hline 21 & 2007-01-04 & FBS34 & 614 & A & $\begin{array}{c}\mathrm{T} \approx 0^{\circ} \mathrm{O} C, 10 \mathrm{~mm} \text { precipitation, } \\
\text { snow depth: } 7 \mathrm{~cm} \text { (on 2007-01-02) and increasing }\end{array}$ & \\
\hline 22 * & $2007-01-21$ & FBS34 & 615 & A & $\begin{array}{c}\mathrm{T} \approx-14 \stackrel{\circ}{\circ} \mathrm{C}, \mathrm{dry}, \\
\text { snow depth: } 28 \mathrm{~cm} \text { (on 2007-01-23) and increasing }\end{array}$ & \\
\hline $23^{*}$ & $2007-02-19$ & FBS34 & 614 & A & $\begin{array}{c}\mathrm{T} \approx-16^{\circ} \mathrm{C}, \mathrm{dry}, \\
\text { snow depth: } 43 \mathrm{~cm} \text { (on 2006-02-21) and increasing }\end{array}$ & \\
\hline 24 & $2007-05-14$ & PLR21 & 606 & A & $\mathrm{T} \approx 7^{\circ} \mathrm{C}, 10 \mathrm{~mm}$ precipitation & \multirow{9}{*}{ Unfr } \\
\hline 25 & $2007-07-07$ & FBD34 & 614 & A & $\begin{array}{l}\mathrm{T} \approx 11^{\circ} \mathrm{C}, 1 \mathrm{~mm} \text { precipitation, } \\
\text { second day of precipitations }\end{array}$ & \\
\hline $26^{*}$ & $2007-07-24$ & FBD34 & 615 & A & $\mathrm{T} \approx 15^{\circ} \mathrm{C},<1 \mathrm{~mm}$ precipitation & \\
\hline $27^{*}$ & $2007-08-05$ & FBD34 & 613 & $\mathrm{~A}$ & $\mathrm{~T} \approx 13^{\circ} \mathrm{C}, \mathrm{dry}$ & \\
\hline 28 & $2007-08-22$ & FBD34 & 614 & $\mathrm{~A}$ & $\mathrm{~T} \approx 9^{\circ} \mathrm{C}$, dry & \\
\hline $29^{*}$ & $2007-09-08$ & FBD34 & 615 & A & $\mathrm{T} \approx 6^{\circ} \mathrm{C}$, dry & \\
\hline 30 * & $2007-09-20$ & FBD34 & 613 & A & $\mathrm{T} \approx 7^{\circ} \stackrel{\circ}{\mathrm{C}}$, dry & \\
\hline 31 & $2007-10-07$ & FBD34 & 614 & A & $\mathrm{T} \approx 6{ }^{\circ} \mathrm{C}, 4 \mathrm{~mm}$ precipitation & \\
\hline 32 & $2007-10-24$ & FBS34 & 615 & A & $\mathrm{T} \approx 2{ }^{\circ} \mathrm{C}$, dry, two days of precipitation before & \\
\hline 33 & 2007-11-05 & FBS34 & 613 & A & $\begin{array}{c}\text { T } \approx 1 \stackrel{\circ}{\circ} \mathrm{C}, 2 \mathrm{~mm} \text { precipitation, } \\
\text { third day of precipitations, } \\
\text { snow depth: } 8 \mathrm{~cm} \text { (on 2007-11-06) }\end{array}$ & Thaw \\
\hline 34 & 2007-11-22 & FBS34 & 614 & A & $\begin{array}{c}\mathrm{T} \approx-3 \stackrel{\circ}{ } \mathrm{C}, \mathrm{dry}, \\
\text { snow depth: } 20 \mathrm{~cm} \text { (on 2007-11-20) and increasing }\end{array}$ & \multirow{2}{*}{$\mathrm{Fr}$} \\
\hline 35 & 2007-12-09 & FBS34 & 615 & A & $\begin{array}{c}\mathrm{T} \approx-9 \stackrel{\circ}{ } \mathrm{C}, \text { dry, } \\
\text { snow depth: } 29 \mathrm{~cm} \text { (on 2007-12-11) }\end{array}$ & \\
\hline 36 & $2007-12-21$ & FBS34 & 613 & A & $\begin{array}{c}T \approx 2 \stackrel{\circ}{ } \mathrm{C} \text {, dry, } \\
\text { snow depth: } 29 \mathrm{~cm} \text { (on 2007-11-19) }\end{array}$ & Thaw \\
\hline 37 & 2008-01-07 & FBS34 & 614 & A & $\begin{array}{c}\mathrm{T} \approx-2^{\circ} \mathrm{\circ} \mathrm{C}, 2 \mathrm{~mm} \text { precipitation, } \\
\text { snow depth: } 38 \mathrm{~cm} \text { (on 2008-01-09) and increasing }\end{array}$ & \multirow{3}{*}{$\mathrm{Fr}$} \\
\hline 38 * & $2008-01-24$ & FBS34 & 615 & A & $\begin{array}{c}\mathrm{T} \approx-4{ }^{\circ} \mathrm{C}, 9 \mathrm{~mm} \text { precipitation, } \\
\text { snow depth: } 56 \mathrm{~cm} \text { (on 2008-01-23) }\end{array}$ & \\
\hline 39 * & $2008-02-05$ & FBS34 & 613 & A & $\begin{array}{c}\mathrm{T} \approx-1^{\circ} \mathrm{\circ} \mathrm{C}, 1 \mathrm{~mm} \text { precipitation, } \\
\text { snow depth: } 80 \mathrm{~cm} \text { (on 2008-02-06) }\end{array}$ & \\
\hline 40 & 2008-03-10 & FBS34 & 615 & A & $\begin{array}{l}\mathrm{T} \approx 1 \stackrel{\circ}{\circ} \mathrm{C}, 3 \mathrm{~mm} \text { precipitation, } \\
\text { fifth day of precipitations, } \\
\text { snow depth: } 81 \mathrm{~cm} \text { (on 2008-03-11) }\end{array}$ & Thaw \\
\hline 41 & $2008-03-22$ & FBS34 & 613 & A & $\begin{array}{c}\mathrm{T} \approx-12 \stackrel{\circ}{ } \mathrm{C}, \text { dry, } \\
\text { snow depth: } 79 \mathrm{~cm} \text { (on 2008-03-19) }\end{array}$ & \multirow{2}{*}{$\mathrm{Fr}$} \\
\hline 42 & 2008-04-08 & FBS34 & 614 & A & $\begin{array}{c}\mathrm{T} \approx-5^{\circ} \mathrm{C},<1 \mathrm{~mm} \text { precipitation, } \\
\text { snow depth: } 65 \mathrm{~cm} \text { (on 2008-04-09) }\end{array}$ & \\
\hline
\end{tabular}


speed has not been reported because of its irrelevance with respect to explaining backscatter signatures. To improve the readability of the environmental conditions, acquisitions were grouped according to a comprehensive definition, i.e., frozen, unfrozen, or thawed conditions. Thaw refers to cases when temperature oscillated around the freezing point on the day of image acquisition. Tables II and III show that the data set over Remningstorp was characterized mostly by unfrozen weather conditions and frequent precipitation. The images over Krycklan were instead often acquired under frozen or thawed conditions.

\section{ANALYSis OF PALSAR Signatures}

For each image, the standwise mean value and standard deviation of the normalized SAR backscattering coefficient $\left(\gamma^{0}\right)$ were computed. For the computation of the standwise measurements from the $\mathrm{K} \& \mathrm{C}$ image strips, the digital standboundary maps were resampled to 50-m pixel spacing. A direct comparison of all measurements is, however, not correct, since the image data sets were characterized by different polarizations, environmental conditions, and viewing geometries. In order to better understand the signatures of the backscatter in relation to each of these factors, subsets were considered that were obtained by selecting those images where all but the factor of interest were constant. More specifically, the backscatter signatures were analyzed in relation to the following conditions:

1) polarization for a given environmental condition and image viewing geometry;

2) environmental conditions for a given polarization and image viewing geometry;

3) image viewing geometry for a given environmental condition and polarization;

4) different spatial resolutions for the same image obtained as path and as $\mathrm{K} \& \mathrm{C}$ strip.

Tree-species composition at stand level was available at Remningstorp; nonetheless, it was decided not to include tree species in the analysis because of the subjective methodology used for the forest-variable estimation. A rigorous analysis of the backscatter signatures in terms of tree species would require that the stands being considered are within a range of stem volumes for which the effect of stem volume itself on the backscatter can be considered negligible. At L-band, only dense forests present a reduced sensitivity of the backscatter. A rough analysis of the tree-species dependence of the backscatter for sets of stands with similar stem volume did not present any significant result also because dense forests were mainly characterized by coniferous species.

\section{A. Polarization Effects}

To investigate the properties of the backscatter for different polarizations, scatterplots were used such as those shown in Fig. 6. The focus was put on the FBD data at $34.3^{\circ}$ because it is the standard imaging configuration at FBD. An assessment of the signatures for the PLR mode was possible only in Remningstorp. Because of the partial coverage, the PLR data set from Krycklan was found to be useless in this context
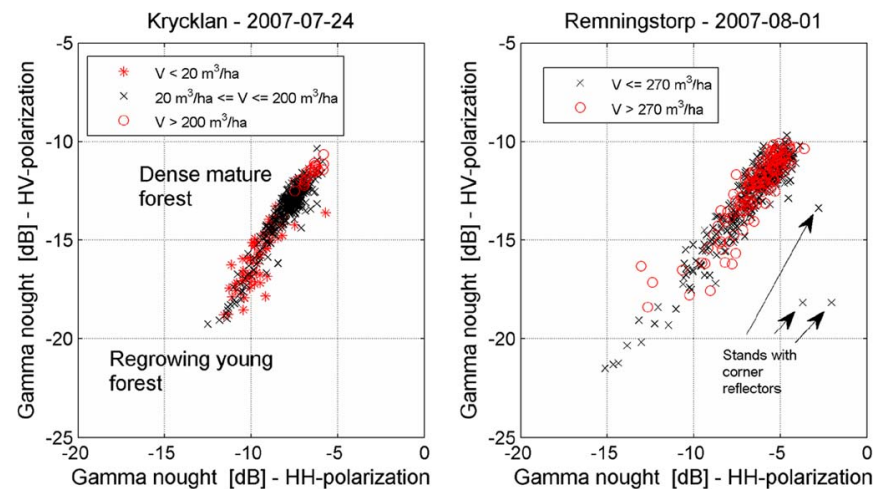

Fig. 6. Comparison of $\mathrm{HH}$ and $\mathrm{HV}$ backscatter (FBD34 imaging configuration) at Krycklan and Remningstorp for dry unfrozen conditions.

TABLE IV

Number of ALOS PALSAR DATA SETS For EACH TEST Site. DATA SPAN From MAY 2006 TO APRIL 2008. EACH DATA SET CONSISTS OF ONE IMAGE IN FBS, TwO IMAGES IN FBD, AND Four IMAGES IN PLR MODE. "PART. COV." INDICATES IMAges Only PARTIAlly COVERING A Test Site

\begin{tabular}{ccc}
\hline ALOS PALSAR & \multicolumn{2}{c}{ Test site } \\
\cline { 2 - 3 } imaging configuration & Remningstorp & Krycklan \\
\hline FBS21 & 2 & 2 \\
\hline FBS34 & 14 & 19 \\
\hline FBS41 & $1+2$ part. cov. & 6 \\
\hline FBD34 & 6 & 3 \\
\hline FBD41 & $2+1$ part. cov. & 0 \\
\hline FBD50 & 12 & 5 part. cov. \\
\hline PLR21 & $39+3$ part. cov. & $37+5$ part. cov.
\end{tabular}

(see Table IV). Since FBD34 and PLR21 have not been acquired during the winter season, the polarization effects on the backscatter could be investigated only for the case of unfrozen environmental conditions.

Fig. 6 shows an example of the behavior of FBD34 $\mathrm{HH}$ and $\mathrm{HV}$ backscatter at the two test sites for images acquired under dry unfrozen conditions. The effect of the environmental conditions will be further discussed in Section IV-B. At both test sites, the backscatter was found to increase from the regrowing young forest to dense mature forest conditions. This is shown by the plot for Krycklan in which the backscatter has been reported for three classes of stem volume $V$ representing regrowing young forest $\left(V<20 \mathrm{~m}^{3} / \mathrm{ha}\right)$, intermediate growth stages ( $V$ between 20 and $200 \mathrm{~m}^{3} /$ ha), and dense mature forest $\left(V>200 \mathrm{~m}^{3} / \mathrm{ha}\right)$. The level of HH backscatter was higher as compared to the HV channel, thus being in agreement with previous investigations as reported in the Introduction. The dynamic range at $\mathrm{HV}$ polarization was greater than at $\mathrm{HH}$ polarization. In Krycklan, the dynamic ranges were $6 \mathrm{~dB}\left(\gamma^{0}\right.$ between -12 and $-6 \mathrm{~dB}$ ) for $\mathrm{HH}$ polarization and $8.5 \mathrm{~dB}$ $\left(\gamma^{0}\right.$ between -19 and $-10.5 \mathrm{~dB}$ ) for $\mathrm{HV}$ polarization. In Remningstorp, the dynamic ranges were similar, being approximately $10 \mathrm{~dB}$. Nonetheless, when excluding from the analysis the few scattered observations at very low backscatter in both channels, the dynamic range was approximately $6.5 \mathrm{~dB}\left(\gamma^{0}\right.$ between -10.5 and $-4 \mathrm{~dB}$ ) for $\mathrm{HH}$ polarization and $8 \mathrm{~dB}$ ( $\gamma^{0}$ between -18 and $-10 \mathrm{~dB}$ ) for HV polarization. At both test sites, the density of the measurements was higher at high 
backscatter because of the large number of stands with dense mature forests. Fig. 6 shows that both HH and HV backscatter of dense mature forests are higher in Remningstorp. To verify whether the higher backscatter was related to the higher stem volumes in Remningstorp, a detailed analysis was performed for the stands with stem volume below the maximum value found in Krycklan, i.e., $270 \mathrm{~m}^{3} /$ ha. The results showed that several stands with stem volume below the threshold had higher backscatter as compared to similar stem volume conditions in Krycklan. Since the ground contribution to the total forest backscatter should be very small, the higher backscatter might be explained as a consequence of a higher density of scattering elements in the forest in Remningstorp. This interpretation is consistent with the fact that, in Remningstorp, the forests are denser as compared to Krycklan.

The scatterplot for Remningstorp also shows that the entire dynamic range was covered by stands with stem volume below $270 \mathrm{~m}^{3} /$ ha. In other words, high backscatter was observed for a large range of stem volumes. Considering that, at this test site, the stem volume reached almost $700 \mathrm{~m}^{3} / \mathrm{ha}$, this result indicates that, for increasing stem volume, the backscatter seemed to loose sensitivity to stem volume. A detailed analysis of the dependence of the backscatter on stem volume is, however, beyond the scope of this paper. The circles at very low backscatter correspond to forest stands with stem volume above $270 \mathrm{~m}^{3} /$ ha. Since it is very improbable that very low backscatter occurs in dense mature forests, these observations should be interpreted as outliers.

An example of the signatures of the polarimetric PLR21 data is shown in Fig. 7, where scatterplots of VV and HV backscatter versus $\mathrm{HH}$ backscatter are reported. A similar scatterplot for $\mathrm{VH}$ versus $\mathrm{HH}$ backscatter has not been included because of the similarity between the HV and the VH backscatter. To be consistent with the analysis of the dual-polarimetric data, a PLR image acquired under dry unfrozen conditions was considered. The HH backscatter was higher as compared to the HV backscatter, whereas the dynamic range of the HV backscatter was larger. Compared to the $\mathrm{HH}$ backscatter, the VV backscatter was lower, and the dynamic range was smaller, thus being in agreement with other observations as reported in the Introduction. More specifically, the HV backscatter varied by approximately $12 \mathrm{~dB}$, with $\gamma^{0}$ between -21 and $-9 \mathrm{~dB}$, when the scattered observations with low backscatter were included. The dynamic range decreased to about $9 \mathrm{~dB}\left(\gamma^{0}\right.$ between -18 and $-9 \mathrm{~dB}$ ) when the few scattered observations with very low backscatter were neglected. For the HH backscatter, the dynamic range was approximately $10\left(\gamma^{0}\right.$ between -13 and $-3 \mathrm{~dB})$ or $7 \mathrm{~dB}\left(\gamma^{0}\right.$ between -10 and $\left.-3 \mathrm{~dB}\right)$, depending on whether the tail of low backscatter stands was considered or not. Finally, for the VV backscatter, the dynamic range was approximately $8\left(\gamma^{0}\right.$ between -13 and $\left.-5 \mathrm{~dB}\right)$ or $6 \mathrm{~dB}\left(\gamma^{0}\right.$ between -11 and $-5 \mathrm{~dB}$ ), depending on whether the tail of low backscatter stands was considered or not.

\section{B. Effects of the Environmental Conditions}

The results shown in Section IV-A were for a specific environmental condition. To get an understanding of the temporal

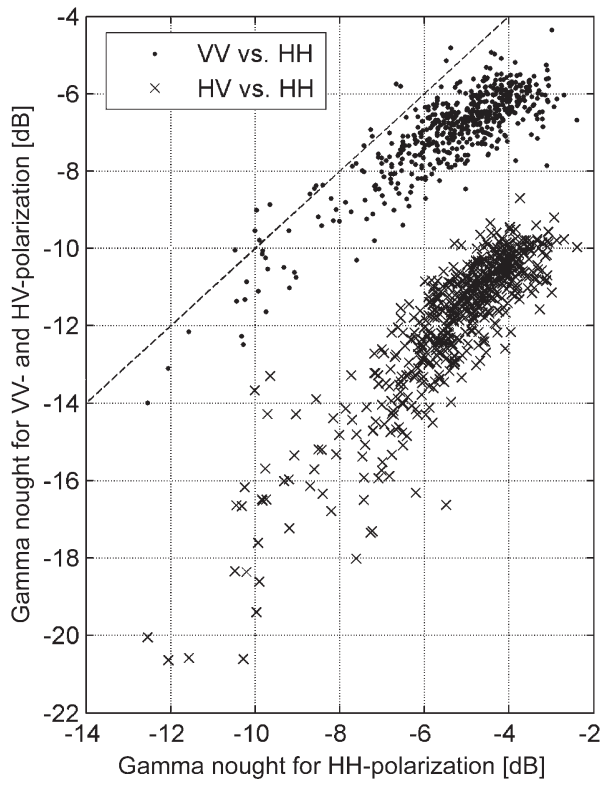

Fig. 7. Scatterplots of VV and HV backscatter versus HH backscatter for the image acquired on June 6, 2007 over Remningstorp in the PLR21 imaging configuration. The dashed line represents the $1: 1$ line.

consistency of the PALSAR backscatter, sets of scatterplots for image pairs acquired with a given polarization and image viewing geometry were analyzed. A full analysis for the typical environmental conditions in Sweden (dry unfrozen, wet unfrozen, frozen, dry thawed, and wet thawed) was possible only for $\mathrm{FB} \mathrm{HH}$ backscatter acquired at $34.3^{\circ}$ look angle. For the FBS21, FBD41, and FBD50 configurations, images were available only for unfrozen conditions. For FBS41, one image was acquired over Krycklan under frozen conditions. For PLR21, one image was acquired over Krycklan under thawing conditions. The analysis focused primarily on the Krycklan data set which was characterized by all environmental conditions typical for Sweden. In Remningstorp, the large majority of the FB HH-polarized images were acquired under unfrozen conditions (see Tables II and III).

1) Krycklan test site: Fig. 8 shows the set of scatterplots for pairs of FB HH-backscatter measurements acquired at $34.3^{\circ}$ in Krycklan. The backscatter of each image is plotted against the backscatter of a common image. This image was acquired on June 27, 2006 when the environmental conditions were dry unfrozen (see Table III). The temporal consistency of the backscatter for unfrozen conditions was high, with correlation coefficients between 0.85 and 0.96 . The dynamic range was 5-6 dB. In most cases, the observations were along the $1: 1$ line. Positive offsets with respect to this line were found for images acquired at the time of much precipitation (September 27, 2006 and October 7, 2007) or after a period of precipitation (October 24, 2007). This can be expected, since the moisture content of the canopy and of the ground surface is higher. The dynamic range decreased because of the slightly higher backscatter in regrowing young forest, where the ground component dominates the total backscatter from the forest stands. Several authors noticed larger dynamic range under dry unfrozen conditions as compared to wet unfrozen conditions which was explained as a consequence of the drier conditions of ground and canopy 


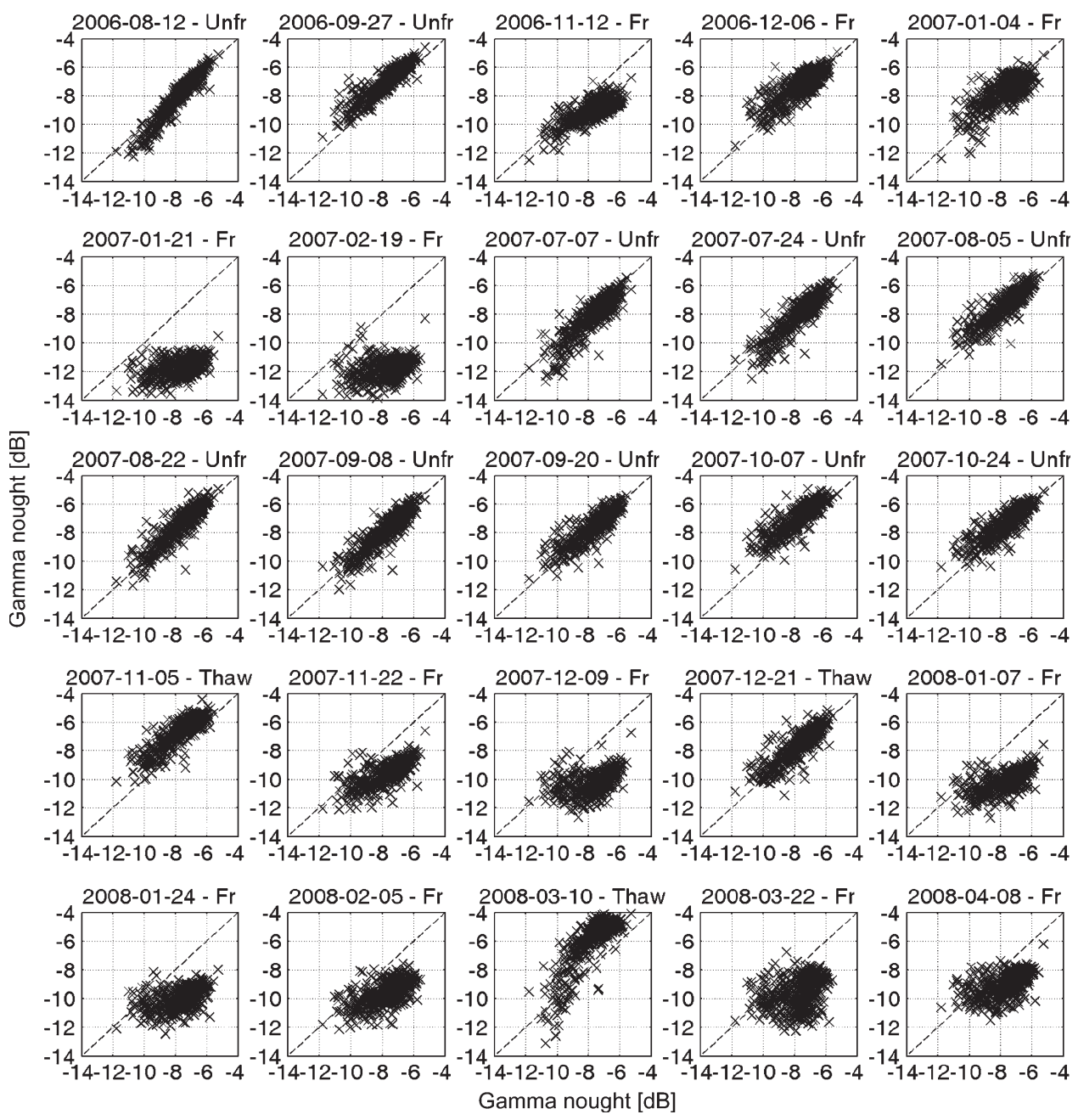

Fig. 8. Scatterplots of FB HH backscatter acquired at $34.3^{\circ}$ in Krycklan with reference to a reference date (June 27, 2006). The backscatter for the reference date is plotted on the horizontal axis. For each image, the acquisition date and the corresponding environmental condition as given in Table III are indicated.

[3], [9], [13], [18]. A negative offset was instead found for two images acquired under practically dry conditions (July 7, 2007 and July 24, 2007). This was more surprising also considering that the deviation from the $1: 1$ line was larger at the lowest backscatter values, for almost bare-soil conditions. The weather statistics did not report any particular difference with respect to other images acquired under dry unfrozen conditions except for wind speed, which was higher $(4-6 \mathrm{~m} / \mathrm{s})$ as compared to the other dates $(<3 \mathrm{~m} / \mathrm{s})$.

For the three images acquired under thawing conditions (November 5, 2007, December 21, 2007, and March 10, 2008), the correlation with respect to the reference data was high (0.83-0.86). However, the behavior was different depending on the kind of thawing conditions on the date of image acquisition. This confirms the indications in [18], where the signatures of the backscatter in Swedish and Siberian forest depended on the temporal dynamics of temperature, snow conditions, and precipitation. A positive offset with respect to the dry unfrozen conditions of the reference image occurred on November 5, 2007 when the thawing event was accompanied by precipitation and there was an 8-cm-thick snow layer. The overall higher backscatter could be due to the wet and thawed conditions of the canopy and to the wet conditions of the ground surface.
This case seems to be similar to what was reported in [4] for Alaskan boreal forest where the higher backscatter under thawing conditions as compared to dry summer conditions was explained as a consequence of the moist biomass of the forest. No offset was found for the December 21, 2007 image, which was acquired under dry conditions with almost 30-cm-deep snow on the ground. The thawed conditions of the trees and the transparency of the snow layer coupled to the frozen conditions of the ground might explain the result. Finally, a nonlinear relationship was found for the March 10, 2008 image with higher backscatter in dense mature forest and lower backscatter in regrowing young forest as compared to the reference image. For dense mature forests, the wet and thawed conditions of the canopy might explain the higher backscatter as for the case of the November 5, 2007 image. For regrowing young forest, the backscatter was related primarily to the scattering within the 80-cm-deep snow layer. A possible explanation for the lower backscatter could be significant absorption within the snow layer. The lack of detailed information on the snow characteristics did not allow for a more detailed interpretation of these backscatter signatures.

The backscatter of images acquired under frozen conditions was less consistent when compared to the reference data set, 

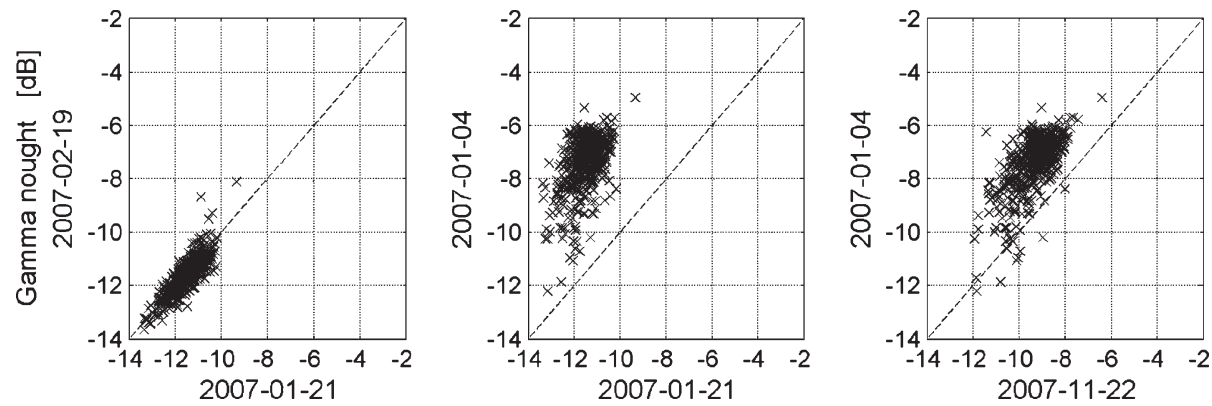

Fig. 9. Scatterplots of FB HH backscatter acquired at $34.3^{\circ}$ in Krycklan for several frozen conditions.

with correlation coefficients between 0.32 and 0.79 . Images acquired when temperature was below $-4{ }^{\circ} \mathrm{C}$ (January 21, 2007, February 19, 2007, December 9, 2007, January 24, 2008, March 22, 2008, and April 8, 2008) were characterized by the lowest correlation (0.32-0.51). Compared to dry unfrozen conditions, the dynamic range was smaller, and the observations were affected by certain spread. Overall, the backscatter was lower, the difference with respect to dry unfrozen conditions being larger in dense mature forest than in regrowing young forest. This could be due to the frozen state of the ground surface and the canopy, which decreased primarily the scattering component from the canopy and, to a minor extent, the scattering from the ground surface. These results agree with the observations of backscatter signatures of forests under frozen and unfrozen conditions in Alaska [4], Finland [13], and Siberia [18]. Images acquired when temperature was between -3 and $0{ }^{\circ} \mathrm{C}$ (November 12, 2006, December 6, 2006, January 4, 2007, November 22, 2007, January 7, 2008, and February 5, 2008) presented correlation coefficients greater than 0.6 and better agreement with the backscatter of dry unfrozen conditions. The first three images had higher correlation (0.74-0.79), and the backscatter showed a significant agreement with the reference image. All three images were acquired during precipitation events. The other three cases were characterized by slightly lower correlation (0.61-0.72) and, with respect to the reference date, showed lower backscatter in dense mature forest but similar backscatter in stands of regrowing young forest. These results tend to indicate that when temperature was closer to the freezing point, the backscatter behavior was probably influenced by the moist canopy and the wetter ground conditions, being similar to what was found for thawed conditions.

Further insight on the backscatter signatures for the case of frozen conditions can be obtained by looking at the scatterplots shown in Fig. 9. On the left-hand side, the backscatter for two images acquired when temperature was much below the freezing point (January 21, 2007 and February 19, 2007) are plotted against each other. The behavior was consistent, and the dynamic range was small $(3 \mathrm{~dB})$, this being a consequence of the frozen state of the canopy that reduce substantially the direct scattering from the canopy and, to a lesser extent, the backscatter from the ground as explained before. The scatterplot in the middle compares the backscatter from an image acquired under frozen conditions with a very low temperature (January 21, 2007) to the backscatter from an image acquired with temperature around the freezing point and precipitation (January 4, 2007). The backscatter for the image acquired on the latter date was higher, particularly in dense mature forests. The higher backscatter and the larger dynamic range (approximately $4 \mathrm{~dB}$ ) were probably due to the thawed conditions of the canopy and the wet conditions of both the canopy and the ground surface. The scatterplot on the right compares two images acquired at temperatures close to the freezing point, one being characterized by precipitation (January 4, 2007), the other by dry conditions (November 22, 2007). The backscatter was overall higher for the image acquired under wet conditions. The dynamic ranges were similar (approximately $4 \mathrm{~dB}$ ). These results confirmed that, under frozen conditions, the backscatter can change depending on both temperature and precipitation. Similar results were found in [4] and [14]. In [14], the authors compared four SIR-C observations under frozen and thawed conditions at a Canadian boreal forest site and found that the backscatter was lowest under frozen conditions when trees and background were frozen. Highest backscatter was found for thawed and wet conditions because of ice and water in the snow cover generating volume scattering. In [4], it was observed that the backscatter increased by $6 \mathrm{~dB}$ from frozen to thawed conditions because the biomass turned from frozen to moist biomass.

For frozen conditions, snow depth did not seem to be particularly related to the backscatter signatures, probably because dry snow is transparent at L-band. However, this does not exclude that the structure of the snow layer and the wetness of the snow might provide more information on the behavior of the backscatter. The lack of more detailed information on the kind of precipitation and on the conditions of the snow layer did not allow further interpretation of the backscatter under frozen conditions.

A more general view on the temporal consistency of the FB HH backscatter at $34.3^{\circ}$ look angle is given in Table V. Table V summarizes the range of the correlation coefficient for different combinations of the environmental conditions. In each case, the highest and lowest correlations, as well as the median value of the correlation, are given. These statistics were preferred, since for several environmental conditions, the number of pairs that could be formed was limited. At the Krycklan test site, the highest overall correlation was obtained when both images were acquired under unfrozen conditions. In addition, the few pairs formed by images acquired under thawing conditions presented high temporal consistency of the measurements with correlation coefficients between 0.80 and 0.94 . Frozen conditions were characterized by temporal variability. For these conditions, higher correlation was obtained 
TABLE V

Correlation COEFFicient Statistics For PAirs of FB HH-BACKSCATter MEASUREMEnTs ACQUiRED AT $34.3^{\circ}$ LOOK ANGLE, AT THE Two TEST Sites

\begin{tabular}{|c|c|c|c|c|c|c|}
\hline & \multicolumn{3}{|c|}{ Krycklan } & \multicolumn{3}{|c|}{ Remningstorp } \\
\hline & \multicolumn{2}{|c|}{ Correlation coefficient } & \multirow{2}{*}{ Number of pairs } & \multicolumn{2}{|c|}{ Correlation coefficient } & \multirow{2}{*}{ Number of pairs } \\
\hline & Min-Max & Median & & Min-Max & Median & \\
\hline Unfr-Unfr & $0.80-0.98$ & 0.90 & 55 & $0.83-0.96$ & 0.89 & 78 \\
\hline Fr-Fr & $0.15-0.90$ & 0.67 & 66 & $0.85-0.92$ & 0.88 & 6 \\
\hline Thaw-Thaw & $0.80-0.94$ & 0.88 & 3 & $0.90-0.90$ & 0.90 & 3 \\
\hline Unfr-Fr & $0.32-0.86$ & 0.65 & 132 & $0.71-0.91$ & 0.82 & 42 \\
\hline Unfr-Thaw & $0.71-0.95$ & 0.88 & 33 & $0.76-0.95$ & 0.87 & 39 \\
\hline Fr-Thaw & $0.24-0.86$ & 0.63 & 36 & $0.82-0.95$ & 0.88 & 12 \\
\hline
\end{tabular}
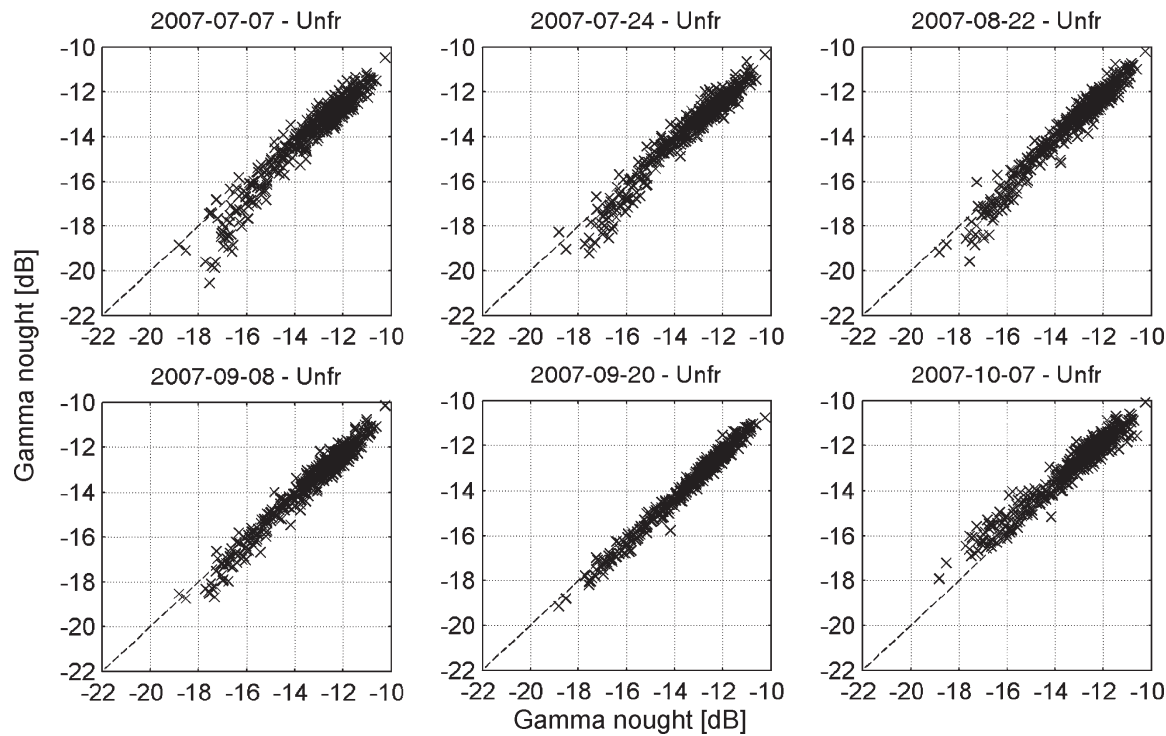

Fig. 10. Scatterplots of FBD34 HV backscatter in Krycklan with reference to a reference date (August 5, 2007). The backscatter from the reference date is plotted on the horizontal axis. For each image, the acquisition date and the corresponding environmental condition as given in Table III are indicated.

either when both images had been acquired when temperature was either very low or close to the freezing point. Images acquired under different environmental conditions presented low correlation when one image had been acquired at frozen conditions with very low temperatures. For all other cases, the correlation coefficient was mostly greater than 0.6.

The analysis of the temporal consistency for cross-polarized backscatter was possible only for unfrozen conditions and for the $34.3^{\circ}$ image viewing geometry. Fig. 10 shows the scatterplots for the HV-backscatter data acquired in FBD34 mode. All scatterplots have the same date as common reference (August 5, 2007) acquired under dry unfrozen conditions. All measurements showed a very high temporal consistency, with correlation between 0.97 and 0.99 . The dynamic range was 7-8 dB. On July 7, 2007, July 24, 2007, August 22, 2007, and October 7, 2007, a small deviation at the lower end of the scatterplot occurred. Similarly to the HH case, the HV backscatter for the first two images was 1-2 dB smaller as compared to the common date. Still, it was unclear what might have caused this decrease, since the environmental conditions were similar except for the higher wind speed for the two images as compared to the reference. The image acquired on October 7, 2007 showed a slightly higher backscatter which could be due to the precipitation that occurred on the day of acquisition (see Table III).

2) Remningstorp test site: In Remningstorp, the seasonal behavior of the FBD34 HH backscatter was similar to Krycklan. The largest dynamic range was observed under unfrozen conditions. Dry unfrozen conditions were characterized by strong temporal consistency and high correlation. Images acquired under thawing conditions presented high consistency with correlation coefficient equal to 0.9. However, it has to be remarked that only three images were identified as having been acquired under thawing conditions (Table V). With respect to unfrozen conditions, the consistency of the backscatter was also high but somewhat variable in time. No significant relationship with specific weather parameter (e.g., amount of precipitation or temperature) could be found.

Under frozen conditions, the smallest dynamic range and overall lower backscatter, in particular, in dense mature forests, were observed. The signatures of the backscatter were consistent, as shown by the high correlation coefficients reported in Table $\mathrm{V}$ for frozen-frozen pairs. The correlation with images acquired under other environmental conditions (i.e., unfrozen-frozen and frozen-thaw) was high in most cases. Compared to Krycklan, the correlation of unfrozen-frozen 

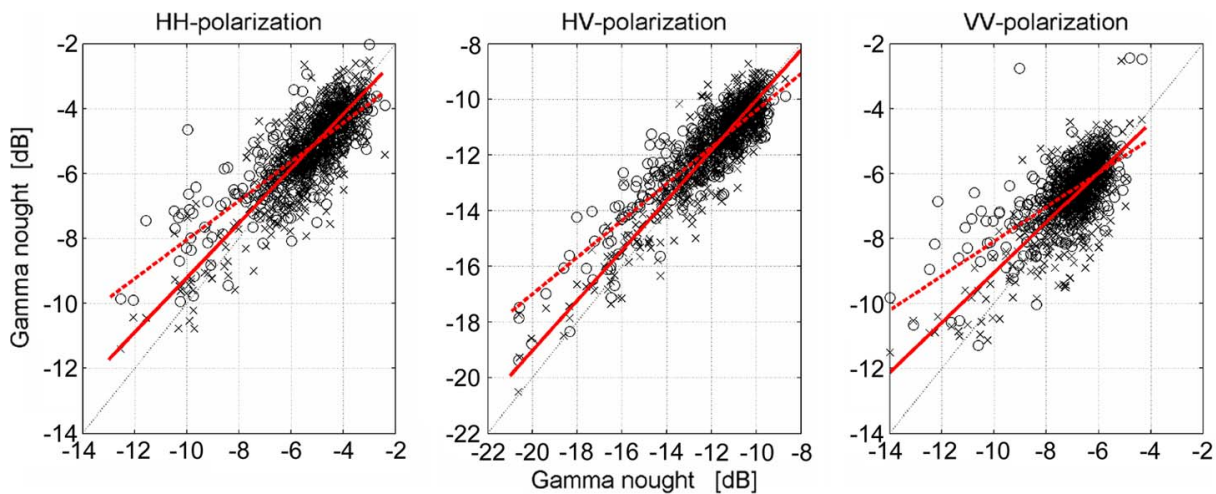

Fig. 11. Scatterplots of HH, HV, and VV backscatter acquired in the PLR mode at $21.5^{\circ}$ look angle. Crosses refer to pairs of measurements acquired under dry unfrozen conditions (June 6, 2007 and June 3, 2006). Circles refer to pairs of measurements acquired under dry (June 6, 2007) and wet conditions (December 4, 2006). On the horizontal axis, data for the common image has been reported.

TABLE VI

List of Images Used in the Assessment of the PALSAR Backscatter Signatures in Relation to Look Angle. All Images Have Been Acquired in FB Mode Except for the June 6, 2007 Image, Which Was Acquired in PLR Mode

\begin{tabular}{ccccc}
\hline \multirow{2}{*}{ Look angle $[\mathrm{deg}]$} & \multicolumn{2}{c}{ Remningstorp } & \multicolumn{2}{c}{ Krycklan } \\
\cline { 2 - 5 } & HH & HV & HH & HV \\
\hline 21.5 & $2006-09-18$ & $2007-06-06$ & $2006-08-27$ & - \\
\hline 34.3 & $2006-07-07$ & $2007-08-01$ & $2006-06-27$ & $2007-08-05$ \\
\hline 41.5 & $2006-08-08$ & $2006-08-08$ & $2006-06-27$ & $2006-07-31$ \\
\hline
\end{tabular}

image pairs was higher. This is very likely a consequence of the smaller number of images acquired under frozen conditions and the rather similar state of these conditions (temperature between $-5{ }^{\circ} \mathrm{C}$ and $-2{ }^{\circ} \mathrm{C}$, thin snow layer, and practically dry conditions). For similar conditions in Krycklan, it was already discussed in Section IV-B1 that the backscatter presented higher correlation for cases with lower temperature and a thicker snow layer. Considering that in southern Sweden temperature is seldom below $-10{ }^{\circ} \mathrm{C}$, the behavior of the backscatter under frozen conditions is well represented by the small data set available for this paper.

FBD34 HV backscatter presented very strong temporal consistency, as already shown in Krycklan, with correlation coefficients between 0.94 and 0.96 for the 15 possible combinations.

The availability of 12 data sets acquired in the PLR21 imaging configuration allowed investigating of the backscatter signatures of all four polarizations simultaneously. Polarimetric data were acquired only under unfrozen conditions (both dry and wet). Fig. 11 shows scatterplots for $\mathrm{HH}, \mathrm{HV}$, and VV backscatter, for two different combinations of unfrozen conditions. Crosses refer to a pair of images both acquired under dry conditions (June 3, 2006 and June 6, 2007). Circles have been used for a pair of images formed by an image acquired under dry conditions (June 6, 2007) and one image acquired under wet conditions (December 4, 2006). To improve the clarity of the plots and identify the main trends of the observations, the linear regression to the observations has been added. It should be remarked that the linear regression has the only meaning of identifying a main trend. Solid lines refer to the dry-dry case; dashed lines refer to the dry-wet case. The backscatter was consistent in the dry-dry case, whereas it increased in regrowing young forest for wet conditions, thus decreasing the dynamic range. The increase of the backscatter and the decrease of the dynamic range were the same regardless of the polarization.

\section{Effects of Image Viewing Geometry}

Forest/non-forest contrast increases for increasing look angle because of the decreasing backscatter from the ground (see, e.g., [37]). Nonetheless, also in dense mature forest, the variation of the scattering mechanisms for changing look angle can cause significant decrease of the total backscatter [4], [36]. For Alaskan forest, it was found that the AIRSAR HV backscatter was constant up to about $40^{\circ}$ and then slightly decreased by $1-2 \mathrm{~dB}$ up to $55^{\circ}$ [4]. Stronger decrease was found for the $\mathrm{HH}$ backscatter. Between $18^{\circ}$ and $55^{\circ}$, the backscatter dropped by approximately $6 \mathrm{~dB}$, with relevant difference below $25^{\circ}$ and above $50^{\circ}$ as compared to the rather constant level between $30^{\circ}$ and $50^{\circ}$.

The availability of several images acquired during 2006 at different look angles allowed the investigating of the sensitivity of the PALSAR backscatter signatures to the image viewing geometry for different growth stages of the forests. The effect of the look angle on the backscatter signatures could be investigated at both test sites, but only for data acquired under unfrozen conditions, since during winter, PALSAR acquires only in the FBS34 mode. In addition, to avoid effects of the environmental conditions, only images acquired under unfrozen and, when possible, dry conditions were considered. Table VI lists the images used in this analysis and the corresponding look angles. To avoid that possible forest cover changes might have influenced the results, the acquisition date of images for each polarization were selected within an as short period of time as possible. All images were acquired in FB mode except for the HV case at $21.5^{\circ}$ for which only data in PLR mode were 

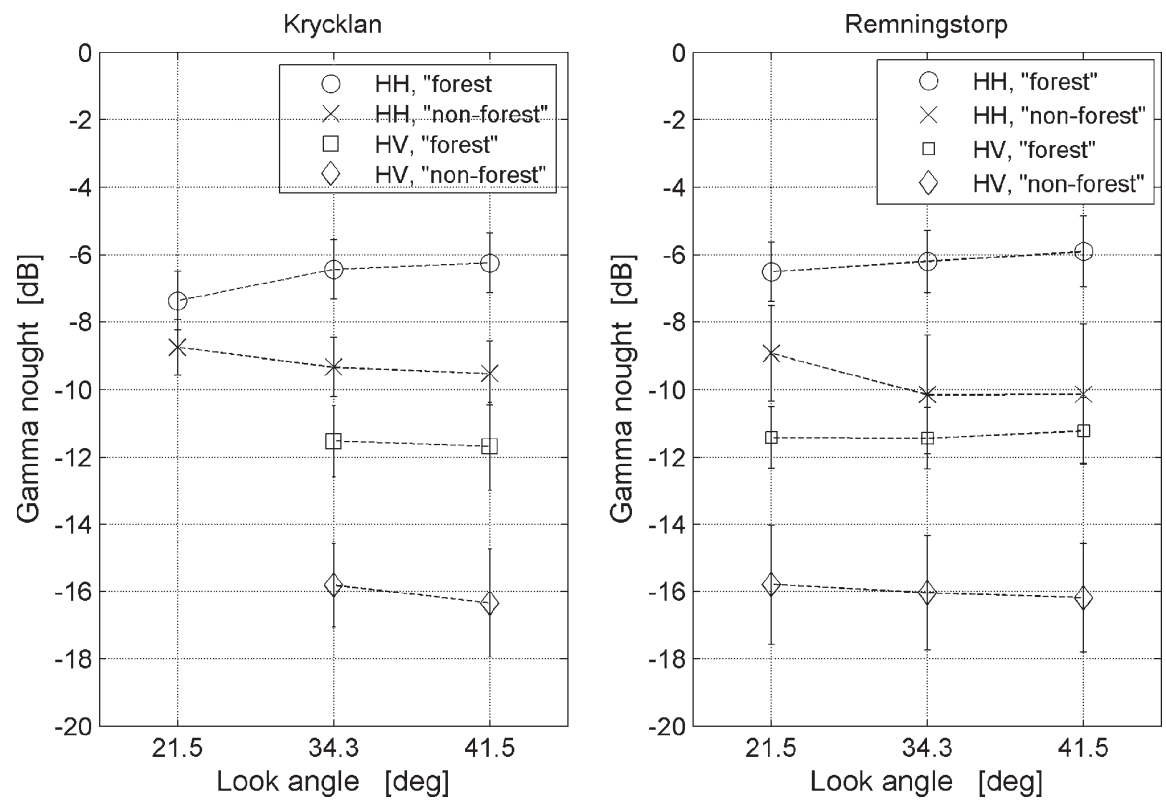

Fig. 12. Mean backscatter and one standard deviation (vertical bars) plotted versus look angle in Krycklan and Remningstorp for (circles) HH backscatter of "forest" stands, (crosses) HH backscatter of "non-forest" stands, (squares) HV backscatter of "forest" stands, and (diamonds) HV backscatter of "non-forest" stands. It is referred to Table VI for the data set of PALSAR images used.

available. For the $50.8^{\circ}$ look angle, two images were available (see Table IV), both were characterized by strong artifacts due presumably to range ambiguities and distorted radiometry. These images were therefore excluded from the analysis.

In Remningstorp, we could investigate the relationship between backscatter and look angle for four different image viewing geometries, owing to the extensive acquisitions during the ALOS Calibration and Validation phase in 2006 [27]. In Krycklan, data have been acquired mostly at $34.3^{\circ}$ and $41.5^{\circ}$ so that the analysis at this test site is somewhat more limited. At each test site, two sets of stands were generated to represent a case with predominantly direct backscatter from the ground and a case with predominantly direct backscatter from the canopy. For simplicity, we will refer to these as the "non-forest" and the "forest" set. In the following, the two sets are described at each test site, and the results of the analysis of the backscatter in relation to look angle are presented. All backscatter measurements were normalized using (1) to a $39^{\circ}$ incidence angle in order to allow intercomparison of measurements taken at different look angles.

1) Krycklan test site: In Krycklan, the standwise stem volume was used to group the stands into the "non-forest" and the "forest" sets. The "non-forest" set was formed by stands with stem volume $<10 \mathrm{~m}^{3} /$ ha $(36$ stands corresponding to $7 \%$ of all stands), whereas the "forest" set was formed by 26 stands (i.e., $5 \%$ of all stands) with stem volume $>200 \mathrm{~m}^{3} /$ ha. The thresholds represented a tradeoff between being consistent with the "forest" and "non-forest" definition of the two sets and including in each set a sufficient number of stands in order to obtain sufficiently accurate estimates of the mean backscatter and the standard deviation. It should be noticed that the uncertainty in the stem volume measurements (see Section II-B) might introduce errors when using the definition of the two sets of stands, which could propagate to the backscatter statistics.
By selecting a sufficient number of stands, we tried limiting this issue.

The plot on the left-hand side in Fig. 12 shows mean and standard deviation of the backscatter for the "forest" and the "non-forest" sets of stands for both the HH and the HV polarizations for increasing look angle. The two upper plots refer to the HH case; the two lower plots refer to the HV case. For both polarizations, the "forest" set showed higher average backscatter than the "non-forest" set. At HH polarization, the backscatter of the "forest" set increased from $21.5^{\circ}$ to $41.5^{\circ}$ by slightly more than $1 \mathrm{~dB}$. The backscatter for the "non-forest" set instead decreased by $1 \mathrm{~dB}$. The backscatter difference between the two sets of stands therefore increased when tending to shallower look angles. On average, the difference increased from about $1.5 \mathrm{~dB}$ to about $3 \mathrm{~dB}$ between $21.5^{\circ}$ and $41.5^{\circ}$. The vertical bars, however, show that the range of backscatter differences is large. Some stands showed similar backscatter (at $21.5^{\circ}$ ), whereas other stands presented a difference greater than $4 \mathrm{~dB}$.

These results can be explained as a consequence of decreasing ground scattering and increasing volume scattering for increasing look angle. For increasing look angle, the backscatter from the stands where ground scattering is dominant ("nonforest" set) decreased. In the "forest" set, the backscatter from the canopy is dominant but there is also a certain contribution from the ground surface through the gaps in the canopy. Since, for increasing look angle, the backscatter from the ground surface decreases, the increase of total backscatter for the dense forests should be related to an increase of volume scattering. The effect of soil moisture and soil roughness, as well as the sensitivity of the backscatter to stem volume, should explain the variability of the backscatter represented by the vertical bars.

For the two look angles at which HV-polarized data were available, the backscatter of the "forest" set was constant, 
TABLE VII

Minimum, Maximum, and Mean Backscatter for the Data Sets Formed by the Stands With Stem Volume Below 10 m ${ }^{3} /$ ha And the $^{2}$

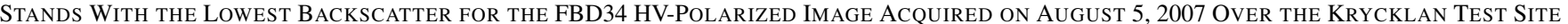

\begin{tabular}{cccc}
\hline Dataset of stands & Minimum $\gamma^{0}[\mathrm{~dB}]$ & Maximum $\gamma^{0}[\mathrm{~dB}]$ & Mean $\gamma^{0}[\mathrm{~dB}]$ \\
\hline$<10 \mathrm{~m}^{3} / \mathrm{ha}(36$ stands) & -17.7 & -13.1 & -15.9 \\
\hline Lowest backscatter (40 stands) & -18.8 & -16.2 & -16.8 \\
\hline
\end{tabular}

whereas it decreased by almost $1 \mathrm{~dB}$ for the "non-forest" set. On average, the backscatter difference between the "forest" and the "non-forest" set increased from 4 to $5 \mathrm{~dB}$. Decreasing backscatter from the ground surface explains the lower backscatter at the shallower look angle. To obtain a constant backscatter in the "forest" set, the volume contribution should have slightly increased in order to counterbalance the decrease of scattering from the ground surface. The vertical bars show a certain variability, $2-3 \mathrm{~dB}$, in particular, at $41.5^{\circ}$. This is probably a consequence of the sensitivity of the backscatter to stem volume, particularly in young regrowing forest. The vertical bars indicate that the overall backscatter difference between dense mature and young regrowing forest conditions could range between 2 and $7 \mathrm{~dB}$. Further discussion on these observations can be found in Section IV-C2 where the trends for Krycklan are compared to the observations from Remningstorp.

The results for the "forest" set shown in the plot on the left-hand side of Fig. 12 did not take into account possible compensation of the backscatter for stands on sloped terrain (see Section III). For different local incidence angles, the radar senses the following conditions: 1) more or less forest floor due to variations in the gap percentage and 2) a larger or smaller canopy. At $\mathrm{HH}$ polarization, both aspects are of importance. At $\mathrm{HV}$ polarization, the ground component is negligible. In [36], an analysis of L-band backscatter at the Lozère test site in France showed that, in old black pine plantations (120140 years), the JERS-1 HH backscatter decreased by about $2 \mathrm{~dB}$ for local incidence angle between $10^{\circ}$ and $80^{\circ}$. For SIR-C $\mathrm{HH}$ and $\mathrm{HV}$ backscatter, the variation between $15^{\circ}$ and $80^{\circ}$ local incidence angle was stronger $(5-7 \mathrm{~dB})$. The range of local incidence angles for the "forest" set in Krycklan was mostly $30^{\circ}-50^{\circ}$ for the configurations at $34.3^{\circ}$ and $41.5^{\circ}$. For such range of angles, the backscatter decreased by less than $1 \mathrm{~dB}$ for the JERS-1 configuration ( $38^{\circ}$ incidence angle at midswath) and less than $2 \mathrm{~dB}$ for the SIR-C configuration $\left(55^{\circ}\right.$ incidence angle at midswath). From these values, it can be inferred that, for the imaging configuration of PALSAR at $34.3^{\circ}$ and $41.5^{\circ}$ look angles, the backscatter should have been corrected by not more than $1 \mathrm{~dB}$, depending on local slope. Hence, the "forest" backscatter values shown in Fig. 12 would have been affected by such correction marginally so that the observations reported in the previous paragraph are still valid. This assumption was confirmed when looking at the backscatter measurements of the "forest" class. No significant trend in terms of local slope could be found, not even for slopes greater than $10^{\circ}$. This result is, however, of relative validity because the number of dense mature forest stands on sloped terrain was very small as compared to the number of stands on flat terrain.

2) Remningstorp test site: In Remningstorp, the "forest" set was formed by the 37 stands with stem volume above
$400 \mathrm{~m}^{3} /$ ha, which corresponded to $7 \%$ of all stands. For the selection of the "non-forest" set of stands, another approach was considered, since in a few stands with stem volume below $10 \mathrm{~m}^{3} /$ ha, the backscatter, at both $\mathrm{HH}$ and $\mathrm{HV}$ polarizations, was high and comparable to the observations in dense forests. This effect is probably due to the uncertainty in the estimation of the stem volume. Since the HV backscatter presents a strong and consistent dependence on forest growth stage, a reasonable assumption for identifying stands forming the "nonforest" set was to use the HV backscatter. More specifically, we selected the 40 stands with the lowest HV backscatter at $34.3^{\circ}$ (corresponding to $7 \%$ of all stands). As additional requirement, the selected stands had to be at least 1 ha large. The assumption that the stands with the lowest HV backscatter would correspond to areas with very low stem volume was verified in Krycklan. Table VII reports the minimum, maximum, and mean backscatter for the two sets of stands in the case of the HV backscatter acquired on August 5, 2007. Most backscatter values of the stands with the lowest $\mathrm{HV}$ backscatter at $34.3^{\circ}$ were within the range of backscatter values for the stands with stem volume below $10 \mathrm{~m}^{3} /$ ha. In addition, the mean value for the 40 stands with the lowest HV backscatter was within the error bars of the stands with stem volumes below $10 \mathrm{~m}^{3} /$ ha (see Fig. 12). Nevertheless, the average backscatter of the 40 stands with lowest HV backscatter was approximately $1 \mathrm{~dB}$ smaller as compared to the value obtained for the 36 stands with stem volume below $10 \mathrm{~m}^{3} / \mathrm{ha}$. This difference might be related to the presence of a vegetation layer in some of the 36 stands with stem volume below $10 \mathrm{~m}^{3} /$ ha. To compensate for the different characteristics, it was decided to add an offset of $1 \mathrm{~dB}$ to the backscatter of the "non-forest" class. Although this correction is not entirely correct because it is highly empirical and the coefficient could depend on polarization and look angle, it allows more insight on the behavior of the backscatter in terms of look angles as compared to the results obtained at Krycklan.

The plot on the right-hand side of Fig. 12 shows mean and standard deviation of the backscatter for the "forest" and the "non-forest" sets of stands for both the HH and the HV polarizations for increasing look angle. The trends from top to bottom refer to the "forest" HH backscatter, the "non-forest" $\mathrm{HH}$ backscatter, the "forest" HV backscatter, and the "nonforest" HV backscatter. At $\mathrm{HH}$ polarization, the trends at the two test sites were in agreement. The backscatter of the "forest" set increased between $21.5^{\circ}$ and $41.5^{\circ}$ by almost $1 \mathrm{~dB}$ on average. The backscatter of the "non-forest" set decreased by about $1.5 \mathrm{~dB}$ on average. The backscatter difference was larger at Remningstorp, being $2.5 \mathrm{~dB}$ for $21.5^{\circ}$ look angle and $4 \mathrm{~dB}$ for $41.5^{\circ}$ look angle. This is very likely a consequence of the different definition of the "non-forest" sets at the two test sites. The vertical bars were rather long for the "non-forest" set of 

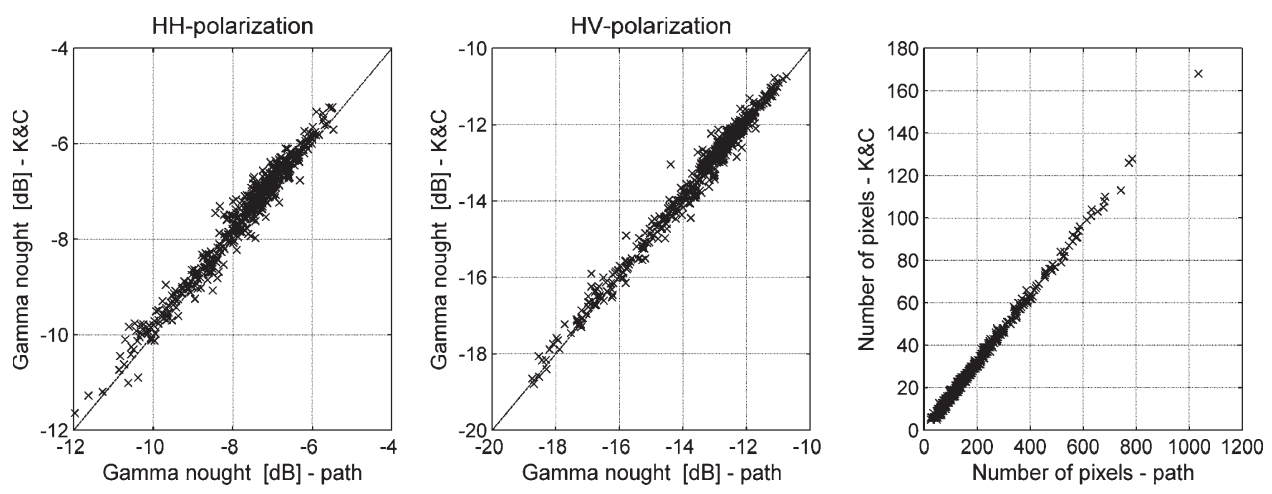

Fig. 13. Scatterplots of standwise (left) HH backscatter, (middle) HV backscatter, and (right) number of pixels for the different spatial resolution of the path and the K\&C strip data. (Test site) Krycklan. The PALSAR image was acquired on September 8, 2007 in FB mode at $34.3^{\circ}$ look angle.

stands (up to $4 \mathrm{~dB}$ at $41.5^{\circ}$ ). Soil moisture, soil roughness, sensitivity to stem volume, and possibly the definition of the "non-forest" class explain the large variability of the signatures. The interpretation of the vertical bars indicates that the difference between the "forest" and the "non-forest" set could range between less than $1 \mathrm{~dB}$ at $21.5^{\circ}$ look angle up to $7 \mathrm{~dB}$ at $34.3^{\circ}$ and $41.5^{\circ}$ look angles.

The HV backscatter of the "forest" set presented a similar behavior as compared to the HH backscatter, and it confirmed the trend found in Krycklan. For the "non-forest" set of stands, the backscatter in the "forest" set was found to be rather constant, whereas it decreased slightly between $21.5^{\circ}$ and $41.5^{\circ}$ by almost $1 \mathrm{~dB}$ on average. The backscatter difference increased on average from $4 \mathrm{~dB}$ at $21.5^{\circ}$ look angle to $5 \mathrm{~dB}$ at $41.5^{\circ}$ look angle. As for the HH backscatter, the "nonforest" set presented long vertical bars ( $3-4 \mathrm{~dB}$ ). These can be explained as a consequence of the sensitivity of the backscatter to stem volume and of the definition of the "non-forest" set. The plot in Fig. 12 shows that backscatter differences between $2 \mathrm{~dB}$ at $21.5^{\circ}$ look angle and $7 \mathrm{~dB}$ at $41.5^{\circ}$ look angle are possible.

\section{Effects of Spatial Resolution}

The availability of both path images at $20-\mathrm{m}$ pixel size and $\mathrm{K} \& \mathrm{C}$ strip images at 50-m pixel size allowed an assessment of the impact of different spatial resolution of the radar images on the backscatter signatures. In Fig. 13, one scatterplot each for $\mathrm{HH}$ and $\mathrm{HV}$ backscatter comparing the backscatter from path data and from K\&C strip data at Krycklan are shown. The consistency between the observations was strong even if the number of pixels on which the standwise backscatter was computed had decreased significantly when going from path data to strip data. The correlation coefficient was 0.98 and 0.99 for $\mathrm{HH}-$ and HV-polarization data, respectively. The mean difference was less than $0.2 \mathrm{~dB}$ with a standard deviation of $0.2 \mathrm{~dB}$. This level was consistent for each data set available at both resolutions in Krycklan. In Remningstorp, the agreement was still strong but the spread was somewhat larger. The correlation coefficient was between 0.8 and 0.9 with mean difference less than $0.5 \mathrm{~dB}$ and standard deviation about $0.3 \mathrm{~dB}$. This slight disagreement between path and K\&C strip backscatter measurements was due to the smaller size of the stands in Remningstorp, which resulted in a degradation of the standwise measurements when considering the lower resolution data, particularly in the smallest stands.

\section{CONCLUSION}

In this paper, ALOS PALSAR L-band backscatter observations of Swedish boreal and hemiboreal forests have been analyzed in relation to polarization, environmental conditions, image viewing geometry, and spatial resolution. The analysis of the backscatter signatures was possible owing to repeated acquisitions for different viewing geometries over two test sites, Remningstorp and Krycklan, located in southern and northern Sweden, respectively.

The analysis of the polarimetric signatures of the backscatter showed that cross-polarized backscatter was more sensitive than copolarized backscatter to the different growth stages, ranging from regrowing young to dense mature forests. For the FBD34 imaging configuration, the dynamic range was similar at the two test sites being $8-9 \mathrm{~dB}$ at $\mathrm{HV}$ polarization and 6-7 $\mathrm{dB}$ at $\mathrm{HH}$ polarization. For the PLR21 imaging configuration, the dynamic range was 6,7 , and $9 \mathrm{~dB}$ at $\mathrm{VV}, \mathrm{HH}$, and $\mathrm{HV}$ polarization, respectively. These values refer to images acquired under dry unfrozen conditions.

For data acquired at $34.3^{\circ}$ in the FB mode and at $21.5^{\circ}$ in the PLR, the dependence of the backscatter on environmental conditions was investigated. Both the copolarized and the crosspolarized backscatter presented strong temporal consistency for images acquired under dry unfrozen conditions. Slight increase of the backscatter in regrowing young forest stands was observed under wet unfrozen conditions, which caused a decrease of the dynamic range by $1-2 \mathrm{~dB}$. The correlation coefficient between images acquired under unfrozen conditions was at least 0.80 for $\mathrm{HH}$ backscatter and 0.94 for HV backscatter. Thawing and frozen conditions occurred primarily for images acquired with the FBS34 (HH polarization) configuration. The backscatter under thawing conditions was similar to the unfrozen case and was rather consistent (correlation coefficient above 0.80 ), although the signatures varied depending on the specific conditions at the time of the thawing event (temperature dynamics, snow-cover properties, precipitation). Under frozen conditions, we observed in Krycklan a different behavior 
of the $\mathrm{HH}$ backscatter depending on the temperature at the time of image acquisition. Compared to unfrozen conditions, the backscatter of both dense mature and regrowing young forests was lower, and the dynamic range was smaller when temperature was below $-4{ }^{\circ} \mathrm{C}$. The signatures of the backscatter for temperatures closer to the freezing point were similar to the unfrozen case. The correlation was, in general, high (above 0.6 ), unless one of the two images forming a pair had been acquired when temperature was several degrees below the freezing point. These observations were confirmed in Remningstorp where three cases of images acquired under frozen conditions were available, with temperatures close to the freezing point. The correlation coefficient of pairs, including one or two images acquired under frozen conditions, was always above 0.7 .

The availability of images acquired at different viewing geometries allowed investigating of the dependence of the forest backscatter on look angle. For this purpose, images acquired in FB mode under unfrozen conditions (preferably dry) were used. To distinguish the signatures of the backscatter for different forest-cover types, two sets of stands, including high stem-volume forest ("forest" set) and regrowing young forest ("non-forest" set), were considered. At both test sites, the HH backscatter in dense mature forests increased slightly between $21.5^{\circ}$ and $41.5^{\circ}$ look angles, whereas the backscatter of regrowing young forests showed a slight decrease. As a consequence, the largest backscatter difference at HH polarization was found at $41.5^{\circ}$. On average, the difference was $3 \mathrm{~dB}$ in Krycklan and $4 \mathrm{~dB}$ in Remningstorp. The variability of the HH-backscatter difference increased for increasing look angle and ranged between 0 and $4 \mathrm{~dB}$ in Krycklan and 0 and $7 \mathrm{~dB}$ in Remningstorp. The HV backscatter of the "forest" set of stands was constant between $21.5^{\circ}$ and $41.5^{\circ}$. The HV backscatter of the "non-forest" set decreased slightly. At both test sites, the backscatter difference between the two sets of stands increased from $21.5^{\circ}$ to $41.5^{\circ}$ from 4 to $5 \mathrm{~dB}$ on average. The variability of the HV-backscatter difference increased for increasing look angle and ranged between 2 and $7 \mathrm{~dB}$ at both test sites. The influence of the look angle on the PALSAR backscatter signatures can therefore be considered to be limited between $21.5^{\circ}$ and $41.5^{\circ}$.

Finally, the availability of images at two different spatial scales $(20$ and $50 \mathrm{~m})$ allowed investigating whether loss of resolution has an impact on the backscatter signatures. In Krycklan, the forest stands were large enough so that no degradation of the standwise backscatter measurements occurred. In Remningstorp, some deviations were observed, due primarily to the smaller stand size.

A summary of the main outcomes from the investigations of a large stack of ALOS PALSAR are therefore as follows.

1) The HV backscatter is more sensitive than $\mathrm{HH}$ and VV backscatter to forest growth conditions.

2) The HV backscatter has a very high temporal consistency under unfrozen conditions.

3) The HH backscatter presents clear seasonal dependences; for unfrozen conditions, the backscatter of regrowing young forest is affected by the wet or dry weather conditions.

4) The sensitivity to forest growth stage increases slightly between $21.5^{\circ}$ and $41.5^{\circ}$ look angles, both at $\mathrm{HH}$ and $\mathrm{HV}$ polarizations.

5) The backscatter signatures do not change significantly between 20- and 50-m pixel size.

While several results were already reported in previous investigations, it is important to remark that the availability of the large stack of ALOS PALSAR images allowed confirming of the indications obtained with sparser data sets from past airborne and spaceborne missions, thus adding consistency to previous conclusions and providing a more general overview on the signatures of L-band backscatter in terms of the factors affecting the backscatter in boreal and hemiboreal forests.

Analysis of the backscatter signatures is important for the development of methods for forest-resource mapping. First indications on the possibility to detect forest changes using PALSAR backscatter were reported in [38] and [39]. The differences of FBD34 HH and HV backscatter between reference forest stands and clear-felled stands were 2.4 and $2.9 \mathrm{~dB}$, respectively. The results reported in this paper indicate that forest change detection seems feasible by using the FB-mode HV backscatter acquired under unfrozen conditions and preferably at shallower look angles because of the strong temporal consistency and the large dynamic range. For the same configuration, HH backscatter from unfrozen conditions also seems useful. HH-polarized backscatter from wintertime images appears less reliable. Preliminary results on forest stemvolume retrieval from PALSAR backscatter in Remningstorp have been presented in [40] and [41]. Backscatter was found to saturate in most cases except for FBS34 HH-backscatter images acquired under frozen conditions. The analysis of the backscatter signatures presented in this paper indicates a certain dependence of the PALSAR backscatter on growth stage, particularly at $34.3^{\circ}$ and $41.5^{\circ}$ look angles. The dependence is also consistent under most environmental conditions. Nonetheless, in this context we did not perform specific investigations on the relationship between backscatter and stem volume. The assessment of the relationship between the PALSAR backscatter and stem volume should be the topic of future investigations.

\section{ACKNOWLEDGMENT}

The authors would like to thank the staff at the Forestry Society's Estate Management Company (Skogssällskapet) for the assistance in the forest operations. They would also like to thank the two anonymous reviewers for their comments. This paper has been undertaken within the framework of the JAXA K\&C Initiative. ALOS PALSAR data have been provided by JAXA EORC within the ALOS K\&C Initiative and the ALOS Calibration and Validation activities. Weather data were provided by the Swedish Meteorological and Hydrological Institute and the Svartberget research station, SLU. The DEMs were obtained from the Swedish National Land Survey. 


\section{REFERENCES}

[1] Å. Rosenqvist, M. Shimada, N. Ito, and M. Watanabe, "ALOS PALSAR: A pathfinder mission for global-scale monitoring of the environment," IEEE Trans. Geosci. Remote Sens., vol. 45, no. 11, pp. 3307-3316, Nov. 2007.

[2] M. C. Dobson, F. T. Ulaby, T. Le Toan, A. Beaudoin, E. S. Kasichke, and N. Christensen, "Dependence of radar backscatter on coniferous forest biomass," IEEE Trans. Geosci. Remote Sens., vol. 30, no. 2, pp. 412-416, Mar. 1992.

[3] K. J. Ranson and G. Sun, "Mapping biomass of a northern forest using multifrequency SAR data," IEEE Trans. Geosci. Remote Sens., vol. 32, no. 2, pp. 388-396, Mar. 1994.

[4] E. Rignot, J. Way, C. Williams, and L. Viereck, "Radar estimates of aboveground biomass in boreal forests of interior Alaska," IEEE Trans. Geosci. Remote Sens., vol. 32, no. 5, pp. 1117-1124, Sep. 1994.

[5] P. A. Harrell, L. L. Bourgeau-Chavez, E. S. Kasischke, N. H. F. French, and N. L. Christensen, Jr., "Sensitivity of ERS-1 and JERS-1 radar data to biomass and stand structure in Alaskan boreal forest," Remote Sens. Environ., vol. 54, no. 3, pp. 247-260, 1995.

[6] M. L. Imhoff, "Radar backscatter and biomass saturation: Ramifications for global biomass inventory," IEEE Trans. Geosci. Remote Sens., vol. 33, no. 2, pp. 511-518, Mar. 1995.

[7] Y. Wang, F. W. Davis, J. M. Melack, E. S. Kasischke, and N. L. Christensen, Jr., "The effects of changes in forest biomass on radar backscatter from tree canopies," Int. J. Remote Sens., vol. 16, no. 3, pp. 503-513, Feb. 1995.

[8] S. M. Yatabe and D. G. Leckie, "Clearcut and forest-type discrimination in satellite SAR imagery," Can. J. Remote Sens., vol. 21, no. 4, pp. 455467, 1995.

[9] P. A. Harrell, E. S. Kasischke, L. L. Bourgeau-Chavez, E. M. Haney, and N. L. Christensen, Jr., "Evaluation of approaches to estimating aboveground biomass in southern pine forests using SIR-C data," Remote Sens. Environ., vol. 59, no. 2, pp. 223-233, 1997.

[10] K. J. Ranson and G. Sun, "An evaluation of AIRSAR and SIR-C/X-SAR images for mapping northern forest attributes in Maine, USA," Remote Sens. Environ., vol. 59, no. 2, pp. 203-222, 1997.

[11] J. R. Baker and A. J. Luckman, "Microwave observations of boreal forests in the NOPEX area of Sweden and a comparison with observations of a temperate plantation in the United Kingdom," Agric. For. Meteorol., vol. 98/99, pp. 389-416, 1999.

[12] J. E. S. Fransson and H. Israelsson, "Estimation of stem volume in boreal forests using ERS-1 C- and JERS-1 L-band SAR data," Int. J. Remote Sens., vol. 20, no. 1, pp. 123-137, Jan. 1999.

[13] J. T. Pulliainen, L. Kurvonen, and M. T. Hallikainen, "Multitemporal behavior of L- and C-band SAR observations of boreal forests," IEEE Trans. Geosci. Remote Sens., vol. 37, no. 2, pp. 927-937, Mar. 1999.

[14] K. J. Ranson and G. Sun, "Effects of environmental conditions on boreal forest classification and biomass estimates with SAR," IEEE Trans. Geosci. Remote Sens., vol. 38, no. 3, pp. 1242-1252, May 2000.

[15] H. Balzter, J. R. Baker, M. Hallikainen, and E. Tomppo, "Retrieval of timber volume and snow water equivalent over a Finnish boreal forest from airborne polarimetric synthetic aperture radar," Int. J. Remote Sens., vol. 23, no. 16, pp. 3185-3208, 2002.

[16] J. Askne, M. Santoro, G. Smith, and J. E. S. Fransson, "Multitemporal repeat-pass SAR interferometry of boreal forests," IEEE Trans. Geosci. Remote Sens., vol. 41, no. 7, pp. 1540-1550, Jul. 2003.

[17] Y. Rauste, "Multi-temporal JERS SAR data in boreal forest biomass mapping," Remote Sens. Environ., vol. 97, no. 2, pp. 263-275, Jul. 2005.

[18] M. Santoro, L. Eriksson, J. Askne, and C. Schmullius, "Assessment of stand-wise stem volume retrieval in boreal forest from JERS-1 L-band SAR backscatter," Int. J. Remote Sens., vol. 27, no. 16, pp. 3425-3454, 2006.

[19] M. Watanabe, M. Shimada, A. Rosenqvist, T. Tadono, M. Matsuoka, S. A. Romshoo, K. Ohta, R. Furuta, K. Nakamura, and T. Moriyama, "Forest structure dependency of the relation between L-band $\sigma^{0}$ and biophysical parameters," IEEE Trans. Geosci. Remote Sens., vol. 44, no. 11, pp. 3154-3165, Nov. 2006.

[20] Y. Wang, J. L. Day, F. W. Davis, and J. M. Melack, "Modeling L-band radar backscatter of Alaskan boreal forest," IEEE Trans. Geosci. Remote Sens., vol. 31, no. 6, pp. 1146-1154, Nov. 1993.

[21] R. Kwok, E. J. M. Rignot, J. Way, A. Freeman, and J. Holt, "Polarization signatures of frozen and thawed forests of varying environmental state," IEEE Trans. Geosci. Remote Sens., vol. 32, no. 2, pp. 371-381, Mar. 1994.
[22] J. Way, E. J. M. Rignot, K. C. McDonald, R. Oren, R. Kwok, G. Bonan, M. C. Dobson, L. A. Viereck, and J. E. Roth, "Evaluating the type and state of Alaska taiga forests with imaging radar for use in ecosystem models," IEEE Trans. Geosci. Remote Sens., vol. 32, no. 2, pp. 353-370, Mar. 1994.

[23] K. J. Ranson, S. Saatchi, and G. Sun, "Boreal forest ecosystem characterization with SIR-C/XSAR," IEEE Trans. Geosci. Remote Sens., vol. 33, no. 4, pp. 867-876, Jul. 1995.

[24] Y. Wang, J. L. Day, and F. W. Davis, "Sensitivity of modeled C- and L-band radar backscatter to ground surface parameters in loblolly pine forest," Remote Sens. Environ., vol. 66, no. 3, pp. 331-342, 1998.

[25] J. J. van Zyl, "The effect of topography on radar scattering from vegetated areas," IEEE Trans. Geosci. Remote Sens., vol. 31, no. 1, pp. 153-160, Jan. 1993

[26] J. Way, J. Paris, E. Kasischke, C. Slaughter, L. Viereck, N. Christensen, M. C. Dobson, F. Ulaby, J. Richards, A. Milne, A. Sieber, F. J. Ahern, D. Simonett, R. Hoffer, M. Imhoff, and J. Weber, "The effect of changing environmental conditions on microwave signatures of forest ecosystems: Preliminary results of the March 1988 Alaskan aircraft SAR experiment," Int. J. Remote Sens., vol. 11, no. 7, pp. 1119-1144, 1990.

[27] L. E. B. Eriksson, G. Sandberg, L. M. H. Ulander, G. Smith-Jonforsen, B. Hallberg, K. Folkesson, J. E. S. Fransson, M. Magnusson, and H. Olsson, "ALOS PALSAR calibration and validation results from Sweden," in Proc. IGARSS, Barcelona, Spain, Jul. 23-27, 2007, pp. 1589-1592.

[28] A. Rosenqvist, M. Shimada, R. Lucas, J. Lowry, P. Paillou, and B. Chapman, The ALOS Kyoto \& Carbon Initiative, Science Plan (v.3.1): JAXA EORC, 2008. [Online]. Available: http://www.eorc.jaxa.jp/ ALOS/kyoto/KC-Science-Plan_v3.1.pdf

[29] M. Magnusson and J. E. S. Fransson, "Evaluation of aerial photointerpretation for estimation of forest stem volume at stand level," in Proc. ForestSat-Operational Tools in Forestry Using Remote Sensing Techniques, Borås, Sweden, May 31-Jun. 3, 2005, pp. 102-106.

[30] E. Næsset, "Estimating timber volume of forest stands using airborne laser scanner data," Remote Sens. Environ., vol. 61, no. 2, pp. 246-253, 1997.

[31] M. Magnusson, J. E. S. Fransson, and J. Holmgren, "Effects on estimation accuracy of forest variables using different pulse density of laser data," For. Sci., vol. 53, no. 6, pp. 619-626, 2007.

[32] M. Shimada, O. Isoguchi, T. Tadono, and K. Isono, "PALSAR radiometric and geometric calibration," IEEE Trans. Geosci. Remote Sens., vol. 47, no. 12, pp. 3915-3932, Dec. 2009.

[33] U. Wegmüller, "Automated terrain corrected SAR geocoding," in Proc. IGARSS, Hamburg, Germany, Jun. 28-Jul. 2, 1999, pp. 1712-1714.

[34] U. Wegmüller, C. Werner, T. Strozzi, and A. Wiesmann, "Automated and precise image registration procedures," in Analysis of Multi-Temporal Remote Sensing Images, vol. 2, L. Bruzzone and P. Smits, Eds. Singapore: World Scientific, 2002, pp. 37-49.

[35] L. M. H. Ulander, "Radiometric slope correction of synthetic-aperture radar images," IEEE Trans. Geosci. Remote Sens., vol. 34, no. 5, pp. 1115-1122, Sep. 1996.

[36] T. Castel, A. Beaudoin, N. Stach, N. Stussi, T. Le Toan, and P. Durand, "Sensitivity of space-borne SAR data to forest parameters over sloping terrain. Theory and experiment.," Int. J. Remote Sens., vol. 22, no. 12, pp. 2351-2376, 2001.

[37] Y. Rauste, "Incidence-angle dependence in forested and non-forested areas in Seasat SAR data," Int. J. Remote Sens., vol. 11, no. 7, pp. 12671276, 1990.

[38] J. E. S. Fransson, M. Magnusson, H. Olsson, L. E. B. Eriksson, G. Sandberg, G. Smith-Jonforsen, and L. M. H. Ulander, "Detection of forest changes using ALOS PALSAR satellite images," in Proc. IGARSS, Barcelona, Spain, Jul. 23-27, 2007, pp. 2330-2333.

[39] J. E. S. Fransson, M. Magnusson, H. Olsson, L. E. B. Eriksson, K. Folkesson, G. Sandberg, M. Santoro, and L. M. H. Ulander, "Detection of clear-cuts using ALOS PALSAR satellite images," in Proc. EUSAR, Friedrichshafen, Germany, Jun. 2-5, 2008, pp. 103-106. CD-ROM.

[40] M. Magnusson, J. E. S. Fransson, L. Eriksson, G. Sandberg, G. Smith-Jonforsen, and L. M. H. Ulander, "Estimation of forest stem volume using ALOS PALSAR satellite images," in Proc. IGARSS, Barcelona, Spain, Jul. 23-27, 2007, pp. 4343-4346.

[41] L. E. B. Eriksson, M. Magnusson, J. E. S. Fransson, G. Sandberg, and L. M. H. Ulander, "Stem volume estimation for boreal forest using ALOS PALSAR," in Proc. 5th Int. Symp. Retrieval Bio- Geophys. Parameters From SAR Data Land Appl., Bari, Italy, Sep. 25-28, 2007. 


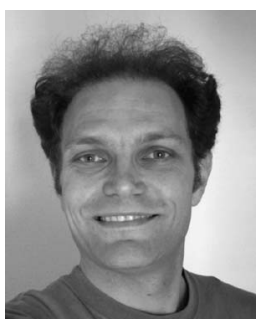

Maurizio Santoro (M'04) received the M.S. degree in aerospace engineering from the University "Federico II," Naples, Italy, in 1998, the Lic.Eng. degree from the Chalmers University of Technology, Göteborg, Sweden, in 2001, and the Ph.D. degree from the Friedrich Schiller University of Jena, Jena, Germany, in 2003.

From 2004 to 2005 , he held a postdoctoral position with the Friedrich Schiller University of Jena. Since 2006, he has been a Project Scientist with Gamma Remote Sensing AG, Gümligen, Switzerland. His main duties include SAR and interferometric SAR data processing and applications of SAR interferometry for land-cover mapping. He is involved as a Principal Investigator and Coinvestigator in several international projects on the use of Earth observation data for land-cover mapping and monitoring. His main research interests include characterization of land cover using SAR and interferometric SAR data and retrieval techniques of forest biophysical parameters from SAR data.

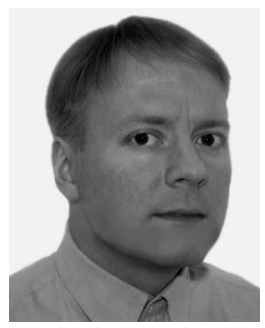

Johan E. S. Fransson (M'02) was born in Karlshamn, Sweden, in 1967. He received the M.Sc. degree in forestry and the Ph.D. degree in forestry remote sensing from the Swedish University of Agricultural Sciences (SLU), Umeå, Sweden, in 1992 and 1999, respectively.

Since 1993, he has been with the Department of Forest Resource Management, SLU, where in 2000 and 2002, he was appointed as Assistant Professor and Associate Professor in forestry remote sensing, respectively, and in 2008, he became the Head. His main research interest includes analysis of SAR images for forestry applications.

Dr. Fransson was the recipient of the International Space University Certificate from the Royal Institute of Technology, Stockholm, Sweden, in 1995, and the award from "Kungliga Skytteanska samfundet" to a younger researcher at SLU in 2002.

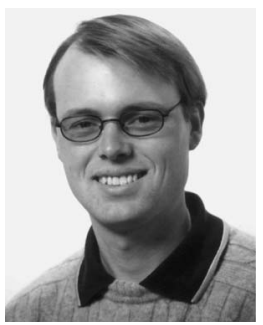

Leif E. B. Eriksson (M'05) received the University Certificate in space technology from Umeå University, Umeå, Sweden, in 1993, the M.S. degree in electrical engineering from the Chalmers University of Technology, Göteborg, Sweden, in 1998, and the Ph.D. degree from the Friedrich Schiller University of Jena, Jena, Germany, in 2004

From 1999 to 2000, he was employed by the Joint Research Centre of the European Commission at the Space Application Institute, Ispra, Italy, where he was working with ERS data for forest and wetland mapping in Siberia. Between 2000 and 2004, he was with the Department of Geoinformatics, Friedrich Schiller University of Jena, Jena, Germany, where he was working with C-and L-band SAR interferometry for forestry applications. Since 2004, he has been with the Department of Radio and Space Science, Chalmers University of Technology, where in 2006, he got the position as Assistant Professor in radar remote sensing. Current research interests include calibration of spaceborne SAR and the use of SAR for forestry and sea-ice applications.

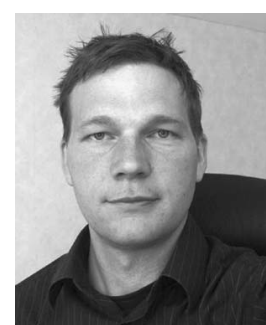

Mattias Magnusson received the M.Sc. degree in forestry and the Ph.D. degree in forestry remote sensing from the Swedish University of Agricultural Sciences, Umeå, Sweden, in 1998 and 2006, respectively.

During 2006 and 2008, he was a Senior Lecturer in geographical information systems with the Swedish University of Agricultural Sciences. Since 2008, he has been a Specialist in forestry and environment with Växjö Stift, Växjö, Sweden. He is the Project Manager of the forest inventory mission. His main research duties include estimation of forest parameters, e.g., stem volume using data from SAR, Lidar, and multispectral satellite images.

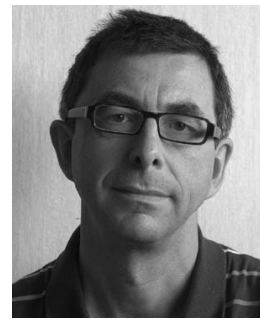

Lars M. H. Ulander (S'86-M'90-SM'04) received the M.Sc. degree in engineering physics and the $\mathrm{Ph} . \mathrm{D}$. degree in electrical and computer engineering from the Chalmers University of Technology, Göteborg, Sweden, in 1985 and 1991, respectively.

Since 1995, he has been with the Swedish Defence Research Agency (FOI), Linköping, Sweden, where he is currently the Director of Research in radar signal processing. He is currently also an Adjunct Professor in radar remote sensing with the Department of Radio and Space Science, Chalmers University of Technology. His research areas are in synthetic aperture radar, electromagnetic scattering models, and remote-sensing applications. He is the author or coauthor of more than 250 professional publications, of which 40 are in peer-reviewed scientific journals. He is the holder of five patents.

Prof. Ulander is a member of the Remote Sensing Committee at the Swedish National Space Board.

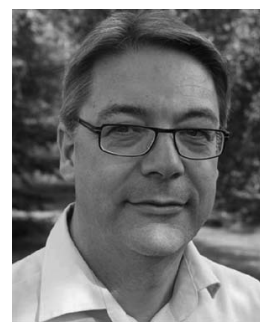

Håkan Olsson received the M.Sc. degree in landscape planning and the Ph.D. degree in forestry remote sensing from the Swedish University of Agricultural Sciences, Umeå, Sweden.

From 1982-1987, he was a Remote Sensing Engineer with the Swedish Space Corporation, Solna, Sweden. Since 1988, he has been with the Faculty of Forest Sciences, Department of Forest Resource Management, Swedish University of Agricultural Sciences, where since 1994, he has been a Professor in forestry remote sensing. 